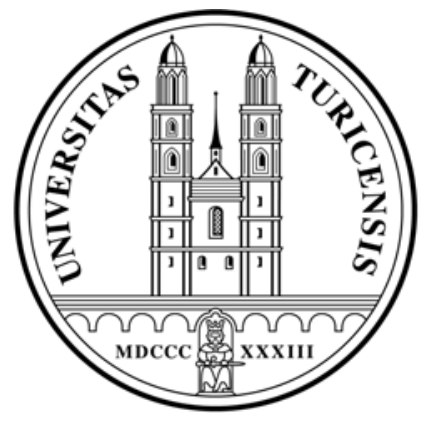

Institute for Empirical Research in Economics

University of Zurich

Working Paper Series

ISSN 1424-0459

Working Paper No. 416

Productivity shocks and aggregate cycles in an estimated endogenous growth model

Jim Malley and Ulrich Woitek

June 2009 


\title{
Productivity shocks and aggregate cycles in an estimated endogenous growth model ${ }^{*}$
}

\author{
Jim Malley \\ University of Glasgow and CESifo \\ Ulrich Woitek \\ University of Zurich and CESifo
}

June 5, 2009

\begin{abstract}
Using a two-sector endogenous growth model, this paper explores how productivity shocks in the goods and human capital producing sectors contribute to explaining aggregate cycles in output, consumption, investment and hours. To contextualize our findings, we also assess whether the human capital model or the standard real business cycle (RBC) model better explains the observed variation in these aggregates. We find that while neither of the workhorse growth models uniformly dominates the other across all variables and forecast horizons, the two-sector model provides a far better fit to the data. Some other key results are first, that Hicks-neutral shocks explain a greater share of output and consumption variation at shorter-forecast horizons whereas human capital productivity innovations dominate at longer ones. Second, the combined explanatory power of the two technology shocks in the human capital model is greater than the Hicks-neutral shock in the RBC model in the medium- and long-term for output and consumption. Finally, the RBC model outperforms the two-sector model with respect to explaining the observed variation in investment and hours.
\end{abstract}

Keywords: endogenous growth, human capital, real business cycles, Bayesian estimation, VAR errors

JEL codes: C11, C52, E32

\footnotetext{
*We would like to thank seminar participants at the Institute for Empirical Research in Economics, University of Zurich, Bodensee Symposium, (May 6-8, 2009) for helpful comments and suggestions. The usual disclaimer applies.
} 


\section{Introduction}

Since the seminal work of Kydland and Prescott (1982) a massive amount of research has been undertaken to better understand the links between technology shocks and business cycles. Likewise the path-breaking research of Romer (1986) and Lucas (1988), stressing the roles of knowledge and human capital accumulation, has led to an enormous body of literature seeking to explain the determinants of endogenous growth. ${ }^{1}$

While the RBC and endogenous growth models differ with respect to their emphasis on the short- and long-run respectively, it is widely recognized that the former model can be viewed as special case of a similarly specified stochastic growth model. For example Jones (2005, p. 806.), using calibrated endogenous growth models, states "[t]he models we study have close analogues in the RBC literature, and hence are a natural first step when moving beyond the standard RBC model. In fact, we interpret the exogenous growth rate of productivity as an endogenous growth rate of human capital. This perspective allows us to compare the strengths of both classes of models using a large number of moments of the joint distribution of macroeconomic time series".

In this spirit and with the aim of better understanding the effects and importance of productivity shocks on business cycles, we econometrically estimate an endogenous growth model incorporating human capital accumulation as well as exogenous productivity shocks in the goods and human capital producing sectors. ${ }^{2}$ When adopting the human capital framework for our purposes, it is important to take into account that the literature has also emphasized the potential role of human capital externalities. This is typically achieved by allowing the return on the human capital of private agents to be increasing in the average stock of human capital in the economy (see e.g. Lucas (1988), Azariadis and Drazen (1990) and Tamura (1991)). The significance of externalities in this context is highlighted by Lucas (2002, p. 6 ), "If ideas are the engine of growth and if an excess of social over private returns is an essential feature of the production of ideas, then we want to go out of our way to introduce external effects into growth theory, not to try to do without them".

\footnotetext{
${ }^{1}$ See the review papers by King and Rebelo (1999) and Rebelo (2005) and references therein on the importance of technology shocks in the real business cycle model. See also Barro and Sala-i-Martin (2004) and Aghion and Howitt (1997) for references on the human capital model.

${ }^{2}$ For recent contributions employing estimated RBC models to examine the effects of disaggregated productivity shocks, see Ireland and Schuh (2008) and Dejong and Ingram (2001).
} 
A vast amount of research has already been directed at quantifying the effect of human capital on growth. For example, studies using reduced-form cross-sectional country or panel regressions unequivocally find a significant link between average years of schooling and growth (see e.g., Barro and Salai-Martin (2004), Barro (2001, 1991), de la Fuente and Domenech (2006)). Other research, based on estimates of the steady-state relationship between growth and human capital, also conclude that human capital contributes either directly or indirectly (via total factor productivity) to growth (see, e.g. Mankiw, Romer and Weil (1992), Benhabib and Spiegel (1994), Bils and Klenow (2000) and Temple (2001)). In stark contrast there is a scarcity of papers which estimate the parameters of the equilibrium conditions in human capital based endogenous growth models. Moreover, to date, there is no significant evidence supporting the existence of aggregate externalities.

Perhaps the dearth of econometric evidence relating to estimates of the structural parameters in these models can be explained by the limited availability of quality human capital data and the previous technical difficulties associated with estimating dynamic stochastic general equilibrium (DSGE) models. However, recent advances in econometric methods and applications go quite some way to mitigating both of these problems. For example, DeJong et al. (2000), Dejong and Ingram (2001), Ireland (2004), FernndezVillaverde, and Rubio-Ramírez (2005), Ireland and Schuh (2008) and Malley and Woitek (2009) provide successful illustrations of estimated RBC or exogenous growth models using both classical and Markov-Chain Monte-Carlo (MCMC) methods. Moreover, using the state-space representation to evaluate the model's likelihood function via the Kalman Filter, helps to avoid problematic measurement issues relating to the human capital data by treating it as an unobservable state variable.

In light of our objectives, we estimate the posterior density function of a prototypical two-sector endogenous growth model using U.S. quarterly data. ${ }^{3}$ Following the hybrid estimation approach of Ireland (2004) we use a firstorder vector autoregressive (VAR) model to describe the movements and comovements of the model's data not explained by the theory. Our estimated model then permits us, using forecast error decompositions, to evaluate the relative importance of total factor productivity (TFP) and human capital productivity in explaining aggregate fluctuations. In conjunction with an estimated RBC model we also assess whether the two-sector model or the $\mathrm{RBC}$ model better explains the observed variation in output, consumption,

\footnotetext{
${ }^{3}$ See Malley and Woitek (2009) for references to the literature on the Bayesian estimation of DSGE models starting with the simulation based methods pioneered by DeJong et al. (2000a,b).
} 
investment and hours and which model provides the best within sample fit. Finally we extract diagnostic information from non-technology shocks to suggest ways to improve the models' ability to capture the dynamics in the data. Addressing these issues using the framework outlined above, constitutes a new contribution to the ongoing literature and debate regarding the effects of innovations to productivity on macroeconomic activity.

Our main results are: (i) in the two-sector model, TFP shocks explain a greater share of output and consumption variation at shorter-forecast horizons whereas human capital productivity innovations dominate at longer ones; (ii) the combined explanatory power of the two technology shocks in the human capital model is greater than the Hicks-neutral shock in the RBC model in the medium- and long-term for output and consumption; (iii) the two-sector model provides a significantly better overall fit to the historical data; however (iv) the RBC model outperforms the human capital model with respect to explaining the observed variation in investment and hours.

\section{Prototype endogenous growth model}

In this section, we solve for the optimal decisions of households and firms relying on the Jones (2005), Lucas (1988) and Tamura (1991) setups. The engine of long-term growth in the Lucas and Tamura models is human capital accumulation in the presence of aggregate externalities. More specifically, externalities imply that the social stock of human capital increases the productivity of individuals' educational choices. The general equilibrium solution consists of a system of dynamic relations, which jointly specify the paths of output, consumption, physical capital, human capital growth, and the fractions of time allocated to work and education. Since the Lucas and Tamura models are well known and effectively represent the industry standard for this class of endogenous growth model (see, e.g. Klenow and RodríguezClare (2005) for a review), the main purpose of this section is simply to fix ideas, notation and variable definitions which will be used in the estimation and analysis which follows.

To facilitate econometric estimation, our deliberately minimal deviations from the Lucas and Tamura setups include: (i) non-zero depreciation rates for human and physical capital; ${ }^{4}$ (ii) the change in human capital is positively

\footnotetext{
${ }^{4}$ Non-zero depreciation rates are not only necessary given that we will be taking the model to the data but also in light of the calibration findings by Jones et al. (2005) who state "A second important finding stems from the fact that the depreciation rate on physical capital is larger than that on human capital. This single asymmetry imparts rich dynamics in the model's response to cyclical shocks".
} 
related to human capital investment via a time-varying stochastic productivity term instead of a constant one; and (iii) TFP is also time-varying and stochastic.

\subsection{Households}

The economy is populated by a large number of identical households indexed by the subscript $h$ and identical firms indexed by the subscript $f$, where $h, f=1,2, \ldots, N_{t}$. The population size, $N_{t}$, evolves at a constant rate $n \geq 1$, so that $N_{t+1}=n N_{t}$, where $N_{0}$ is given. Each household's preferences are given by the following time-separable utility function:

$$
E_{0} \sum_{t=0}^{\infty} \beta^{t} U\left(C_{t}^{h}\right)
$$

where $E_{0}$ is the conditional expectations operator; $C_{t}^{h}$ is consumption of household $h$ at time $t$; and $0<\beta<1$ is the discount rate. The instantaneous utility function is increasing, concave and satisfies the Inada conditions. We use the $C R R A$ form for utility:

$$
U_{t}=\frac{\left(C_{t}^{h}\right)^{1-\sigma}}{1-\sigma}
$$

where, $1 / \sigma(\sigma>1)$ is the inter-temporal elasticity of substitution of consumption.

Each household $h$ saves in the form of investment, $I_{t}^{h}$, and receives interest income, $r_{t} K_{t}^{h}$, where $r_{t}$ is the return to capital and $K_{t}^{h}$ is the beginning-ofperiod private capital stock. The household has one unit of time in each period $t$, which is allocated between work, $u_{t}^{h}$, and education, $e_{t}^{h}$, so that ${ }^{5}$

$$
u_{t}^{h}+e_{t}^{h}=1 \text {. }
$$

A household with a stock of human capital, $H_{t}^{h}$ receives labor income, $w_{t} u_{t}^{h} H_{t}^{h}$, where $w_{t}$ is the wage rate and $u_{t}^{h} H_{t}^{h}$ is $h$ 's effective labor. ${ }^{6}$ Finally, each household receives dividends paid by firms, $\Pi_{t}^{h}$. Accordingly, the budget constraint of each household is:

$$
C_{t}^{h}+I_{t}^{h}=r_{t} K_{t}^{h}+w_{t} u_{t}^{h} H_{t}^{h}+\Pi_{t}^{h} .
$$

\footnotetext{
${ }^{5}$ This formulation implies that leisure time is constant and exogenous.

${ }^{6}$ Note that human capital, $H_{t} \equiv L_{t} H_{t}^{q}$, is the product of the quantity of workers $L_{t}$ and the quality or human capital per worker, $H_{t}^{q}$. Following Lucas (1988), it is assumed that $L_{t}$ and $H_{t}^{q}$ are perfect substitutes so that only $H_{t}$ matters for production.
} 
Each household's physical and human evolve according to:

$$
K_{t+1}^{h}=\left(1-\delta^{k}\right) K_{t}^{h}+I_{t}^{h}
$$

and

$$
H_{t+1}^{h}=\left(1-\delta^{h}\right) H_{t}^{h}+B_{t}\left(e_{t}^{h} H_{t}^{h}\right)^{\theta}\left(\bar{H}_{t}\right)^{1-\theta}
$$

where, $0 \leq \delta^{k}, \delta^{h} \leq 1$ are constant depreciation rates on private physical and human capital respectively. The second term on the r.h.s. of (6), consisting of three multiplicative arguments, can be interpreted as the quantity of "new" human capital created at time period $t .{ }^{7}$ This term is comprised of the following arguments: (i) $B_{t}$ represents human capital productivity; (ii) $\left(e_{t}^{h} H_{t}^{h}\right)$ is $h^{\prime} s$ effective human capital; (iii) $\bar{H}_{t}$ is the average (per household) human capital stock in the economy; and (iv) the parameters $0<\theta,(1-\theta)$ capture the efficiency of household human capital and the aggregate human capital externality respectively. ${ }^{8}$

Households act competitively by taking market prices and aggregate outcomes as given. Thus, each household chooses $\left\{C_{t}^{h}, u_{t}^{h}, e_{t}^{h}, I_{t}^{h}, K_{t+1}^{h}, H_{t+1}^{h}\right\}_{t=0}^{\infty}$ to maximize (1) subject to (3)-(6), and initial conditions for the two capital stocks and the two productivity terms.

The familiar static optimality condition for consumption, $C_{t}^{h}$, is

$$
\Lambda_{t}^{a}=\left(C_{t}^{h}\right)^{-\sigma}
$$

and states that the shadow price associated with (4), $\Lambda_{t}^{a}$, is equal to the marginal value of consumption at time $t$.

The Euler-relation for private physical capital, $K_{t+1}^{h}$, is given by

$$
\Lambda_{t}^{a}=\beta E_{t}\left[\Lambda_{t+1}^{a}\left(r_{t+1}+1-\delta^{k}\right)\right]
$$

and says that marginal cost of forgone consumption at time $t$ is equal to the expected marginal benefit of discounted $t+1$ returns derived from investing in one unit of physical capital at time $t$.

\footnotetext{
${ }^{7}$ The Lucas (1988) physical and human capital relations maintain when $\delta^{k}=\delta^{h}=0$ and $B_{t}=B$ and $\theta=1$.

${ }^{8}$ The assumption that individual human capital accumulation is an increasing function of the per capita level of economy-wide human capital captures the idea that the existing know-how of the economy provides an external positive effect. Equivalently it can be thought of as a learning-by-doing effect as discussed in Romer (1986). Examples of other papers which use the per capita level of aggregate human capital in either the goods or human capital production functions include Lucas (1988), Azariadis and Drazen, (1990), Tamura (1991).
} 
The static optimality condition for time spent on education, $e_{t}^{h}$, can be written as

$$
\Lambda_{t}^{b}=\frac{\Lambda_{t}^{a} w_{t} H_{t}^{h}}{B_{t} \theta\left(e_{t}^{h}\right)^{\theta-1}\left(H_{t}^{h}\right)^{\theta}\left(\bar{H}_{t}\right)^{1-\theta}}
$$

and implies that the shadow price associated with (6), $\Lambda_{t}^{b}$, is equal to the marginal value of education at time $t$. In other words, this value is equal to the ratio of the marginal cost to the marginal return to time spent in education.

The Euler-equation for private human capital, $H_{t+1}^{h}$, is

$$
\begin{aligned}
\Lambda_{t}^{b}= & E_{t} \beta \Lambda_{t+1}^{a} w_{t+1}\left(1-e_{t+1}^{h}\right)+ \\
& E_{t} \beta \Lambda_{t+1}^{b}\left[1-\delta^{h}+B_{t+1} \theta\left(e_{t+1}^{h}\right)^{\theta}\left(H_{t+1}^{h}\right)^{\theta-1}\left(\bar{H}_{t+1}\right)^{1-\theta}\right]
\end{aligned}
$$

and maintains that the marginal cost of forgone labor income at time $t$ is equal to the marginal benefit of $t+1$ returns to working plus the marginal $t+1$ returns to investing in one unit of human capital at time $t$.

\section{$2.2 \quad$ Firm's problem}

To produce its homogenous final product, $Y_{t}^{f}$, each firm employs private physical capital, $K_{t}^{f}$ and effective labor, $u_{t}^{f} H_{t}^{f}$. Thus, the production function of each firm is:

$$
Y_{t}^{f}=A_{t}\left(K_{t}^{f}\right)^{\alpha}\left(u_{t}^{f} H_{t}^{f}\right)^{1-\alpha}
$$

where $A_{t}$ represents the level of Hicks-neutral neutral technology available to all firms, $0<\alpha<1$ and $(1-\alpha)$ are the efficiency of private capital and effective labor respectively.

Firms act competitively by taking prices and aggregate outcomes as given. Accordingly, subject to (11), each firm chooses $K_{t}^{f}$ and $u_{t}^{f} H_{t}^{f}$ to maximize a series of static profit functions,

$$
\Pi_{t}^{f}=Y_{t}^{f}-r_{t} K_{t}^{f}-w_{t} u_{t}^{f} H_{t}^{f} .
$$

The resulting familiar first-order conditions state that the firm will hire labor until the marginal product of effective labor is equal to the wage rate, $w_{t}$, and will rent capital until the marginal product of physical capital is equal to the rental rate, $r_{t}$, i.e.

$$
\frac{(1-\alpha) Y_{t}^{f}}{u_{t}^{f} H_{t}^{f}}=w_{t}
$$


and

$$
\frac{\alpha Y_{t}^{f}}{K_{t}^{f}}=r_{t} .
$$

Finally note that, given the assumption of constant returns to scale in production at the firm level, factor payments exhaust the value of output, i.e. $Y_{t}^{f}=r_{t} K_{t}^{f}+w_{t} u_{t}^{f} H_{t}^{f}$, implying no economic profits are earned.

\subsection{Decentralized competitive equilibrium (DCE)}

The DCE is defined when (i) households and firms optimize, as above, taking prices and aggregate outcomes as given; (ii) all constraints are satisfied; and (iii) all markets clear, i.e. $\sum K_{t}^{h}=\sum K_{t}^{f}, \sum\left(1-e_{t}^{h}\right) H_{t}^{h}=\sum u_{t}^{f} H^{f}, \sum \Pi_{t}^{h}=$ $\sum \Pi_{t}^{f}=0$. Given the $N_{t}$ identical households at time period $t$ and also $N_{t}$ identical firms, economy wide magnitudes are denoted $X_{t}=N_{t} X_{t}^{h}=$ $N_{t} X_{t}^{f}$. Since human capital is the engine of long-run endogenous growth, we transform variables to make them stationary, e.g. we first define per capita quantities for any variable $X$ as $\bar{X}_{t} \equiv X_{t} / N_{t}$, where $X_{t} \equiv\left(Y_{t}, C_{t}, I_{t}, K_{t}, H_{t}\right)$ and then express these as shares of per capita human capital, e.g. $x_{t} \equiv$ $\bar{X}_{t} / \bar{H}_{t}$. Finally, the gross human capital growth rate is defined as $\gamma_{t} \equiv$ $\bar{H}_{t+1} / \bar{H}_{t}$. Using this notation and substituting out prices, $\left\{r_{t}, w_{t}\right\}_{t=0}^{\infty}$, we obtain the following stationary $D C E$ :

$$
\begin{aligned}
y_{t}= & c_{t}+n \gamma_{t} k_{t+1}-\left(1-\delta^{k}\right) k_{t} \\
y_{t}= & A_{t}\left(k_{t}\right)^{\alpha}\left(1-e_{t}\right)^{(1-\alpha)} \\
n \gamma_{t}= & 1-\delta^{h}+B_{t}\left(e_{t}\right)^{\theta} \\
\lambda_{t}^{a}= & \left(c_{t}\right)^{-\sigma} \\
\lambda_{t}^{a}= & \beta\left(\gamma_{t}\right)^{-\sigma} E_{t}\left[\lambda_{t+1}^{a}\left(\alpha \frac{y_{t+1}}{k_{t+1}}+1-\delta^{k}\right)\right] \\
\lambda_{t}^{b}= & \frac{\left(c_{t}\right)^{-\sigma}(1-\alpha) y_{t}}{B_{t}\left(1-e_{t}\right) \theta\left(e_{t}\right)^{\theta-1}} \\
\lambda_{t}^{b}= & \beta\left(\gamma_{t}\right)^{-\sigma}\left\{E_{t}\left[\left(c_{t+1}\right)^{-\sigma}(1-\alpha) y_{t+1}\right]+\right. \\
& \left.E_{t} \lambda_{t+1}^{b}\left[\left(1-\delta^{h}\right)+B_{t+1} \theta\left(e_{t+1}\right)^{\theta}\right]\right\}
\end{aligned}
$$

where $\lambda_{t}^{a}$ and $\lambda_{t}^{b}$ are the transformed shadow prices associated with (4) and (6) respectively in the household's problem. ${ }^{9}$ Therefore, the stationary DCE

\footnotetext{
${ }^{9}$ Note that $\lambda_{t}^{a}=\Lambda_{t}^{a} / \bar{H}_{t}^{-\sigma}$ and $\lambda_{t}^{b}=\Lambda_{t}^{b} / \bar{H}_{t}^{-\sigma}$ where $h$-superscripts have been omitted since we are in a symmetric equilibrium.
} 
is summarized by the above system of seven equations in the paths of the following seven variables: $\left(\gamma_{t}, y_{t}, c_{t}, e_{t}, k_{t+1}, \lambda_{t}^{a}, \lambda_{t}^{b}\right)$ given the paths of the exogenously set stationary AR processes whose motion is defined below.

\subsection{Processes for productivity}

Given the above set-up, Hicks-neutral productivity, $A_{t}$, and human capital productivity, $B_{t}$, are stochastic. Following the usual practice in the RBC literature,${ }^{10}$ we assume that each follows an $\operatorname{AR}(1)$ process:

$$
\begin{aligned}
& A_{t}=A^{\left(1-\rho^{a}\right)} A_{t-1}^{\rho^{a}} e^{\varepsilon_{t}^{a}} \\
& B_{t}=B^{\left(1-\rho^{b}\right)} B_{t-1}^{\rho^{b}} e^{\varepsilon_{t}^{b}}
\end{aligned}
$$

where $A, B>0$ are constants, $0<\rho^{a}, \rho^{b}<1$ are the autoregressive parameters and $\varepsilon_{t}^{a}, \varepsilon_{t}^{b}$ are independent and identically distributed random shocks with zero means and variances equal to $\sigma_{a}^{2}$ and $\sigma_{b}^{2}$ respectively.

Innovations to TFP affect the efficiency of both capital and effective labor, whereas shocks to human capital productivity are purely labor augmenting. DeJong and Ingram (2001, p. 541-42) argue that $B_{t}$ can be thought of as "[...] an exogenous shock that shifts the efficiency with which hours are transformed into human capital. Examples of a negative shock are the creation of a new computer operating system that is more difficult to learn than the previous system and a decrease in funding for government-sponsored training programs. A positive shock could be a technological improvement in employee training methods".

\subsection{Model solution}

Following Klein (2000), we obtain the solution of the linearized stationary $\mathrm{DCE}^{11}$ in state space form

$$
\begin{aligned}
\boldsymbol{y}_{t} & =\tilde{\mathbf{Z}}_{t} \boldsymbol{\delta}_{\boldsymbol{t}} \\
\boldsymbol{\delta}_{t+1} & =\tilde{\mathbf{T}} \boldsymbol{\delta}_{t}+\tilde{\boldsymbol{R}} \boldsymbol{\epsilon}_{t+1}
\end{aligned}
$$

where $\mathbf{y}_{t}=\left[\begin{array}{lll}\hat{y}_{t} & \hat{c}_{t} & \hat{u}_{t}\end{array}\right]^{\prime} ; \boldsymbol{\delta}_{t}=\left[\begin{array}{lll}\hat{k}_{t} & \hat{a}_{t} & \hat{b}_{t}\end{array}\right]^{\prime} ;$ for any variable $x_{t}, \hat{x}_{t}=\ln \left(x_{t} / x\right)$; $x$ is the model-consistent steady-state value of $x_{t} ; \hat{y}_{t}$ is the control variable; $\hat{c}_{t}$ and $\hat{u}_{t}$ are jump variables; $\hat{k}_{t}$ is the state variable; $\hat{a}_{t}$ and $\hat{b}_{t}$ are the two

\footnotetext{
${ }^{10}$ See, e.g. Kim and Lee (2007), DeJong and Ingram (2001) and Perli and Sakellaris (1998) for similar setups for the two productivity processes.

${ }^{11}$ See the Appendix for details.
} 
exogenous processes; $\tilde{\mathbf{Z}}$ and $\tilde{\mathbf{T}}$ are matrices containing various convolutions of the model's parameter (see Appendix Table 1); and $\tilde{\mathbf{R}}_{t}$ is a matrix of zeros and ones controlling two productivity shocks in the vector $\boldsymbol{\epsilon}_{t+1}$.

\section{Econometric Setup}

In this section, following Ireland (2004), we apply the hybrid approach to model estimation by adding $\operatorname{VAR}(1)$ measurement/specification errors to (17). ${ }^{12}$ We calculate the model's likelihood function which requires application of the Kalman filter given that the human and physical capital stocks, both types of technology, and the measurement/specification errors are treated as unobservables.

\section{1 $\quad \operatorname{VAR}(1)$ setup}

Adding an $n$-dimensional (where $n$ refers to the number of measurement equations) VAR(1) error block

$$
\boldsymbol{\mu}_{t+1}=\mathbf{A} \boldsymbol{\mu}_{t}+\boldsymbol{\nu}_{t+1}, \quad \boldsymbol{\nu}_{t+1} \sim N(\mathbf{0}, \mathbf{\Sigma})
$$

to (17) as in Ireland (2004) yields the following state-space representation

$$
\begin{aligned}
\mathbf{y}_{t} & =\left(\begin{array}{ll}
\tilde{\mathbf{Z}} & \mathbf{I}_{n}
\end{array}\right)\left(\begin{array}{c}
\boldsymbol{\delta}_{t} \\
\boldsymbol{\mu}_{t}
\end{array}\right)=\mathbf{Z} \boldsymbol{\alpha}_{t} \\
\boldsymbol{\alpha}_{t+1} & =\left(\begin{array}{cc}
\tilde{\mathbf{T}} & \mathbf{0}_{n \times n} \\
\mathbf{0}_{n \times n} & \mathbf{A}
\end{array}\right) \boldsymbol{\alpha}_{t}+\left(\begin{array}{cc}
\tilde{\mathbf{R}} & \mathbf{0}_{n \times n} \\
\mathbf{0}_{n \times 2} & \mathbf{I}_{n}
\end{array}\right)\left(\begin{array}{c}
\boldsymbol{\epsilon}_{t+1} \\
\boldsymbol{\nu}_{t+1}
\end{array}\right) \\
& =\mathbf{T} \boldsymbol{\alpha}_{t}+\mathbf{R} \boldsymbol{\eta}_{t+1}
\end{aligned}
$$

with

$$
\boldsymbol{\eta}_{t+1} \sim N(\mathbf{0}, \mathbf{Q}), \quad \mathbf{Q}=\left(\begin{array}{ccc}
\sigma_{a}^{2} & 0 & \mathbf{0}_{1 \times n} \\
0 & \sigma_{b}^{2} & \mathbf{0}_{1 \times n} \\
\mathbf{0}_{n \times 1} & \mathbf{0}_{n \times 1} & \boldsymbol{\Sigma}
\end{array}\right)
$$

\footnotetext{
${ }^{12}$ For further details on the hybrid approach to estimation using VAR(1) errors, see Ireland (2004). Following Malley and Woitek (2009) we also considered adding VARMA(1,1) errors to the economic model. However, the logmarginal likelihood difference test of the $\operatorname{VAR}(1)$ versus the VARMA(1,1) yielded a value of 317.27 suggesting a much better within sample fit for the former specification. The Appendix contains details on how we calculate this test.
} 


\subsection{Kalman filter}

For given initial estimates of the state vector, $\mathbf{a}_{0}$, i.e. $\mathbf{a}_{0}=E\left(\boldsymbol{\alpha}_{0}\right)$ and the covariance matrix, $\mathbf{P}_{0}$, the filter consists of the following steps:

1. prediction step

$$
\begin{aligned}
\mathbf{a}_{t \mid t-1} & =\mathbf{T} \mathbf{a}_{t-1} ; \\
\mathbf{P}_{t \mid t-1} & =\mathbf{T} \mathbf{P}_{t-1} \mathbf{T}^{\prime}+R Q \mathbf{R}^{\prime}
\end{aligned}
$$

2. updating step

$$
\begin{aligned}
\boldsymbol{v}_{t} & =\mathbf{y}_{t}-\mathbf{Z} \mathbf{a}_{t \mid t-1} \\
\mathbf{F}_{t} & =\mathbf{Z} \mathbf{P}_{t \mid t-1} \mathbf{Z}^{\prime} \\
\mathbf{K}_{t} & =\mathbf{T} \mathbf{P}_{t \mid t-1} \mathbf{Z}^{\prime} \mathbf{F}_{t}^{-1} \\
\mathbf{a}_{t} & =\mathbf{T} \mathbf{a}_{t \mid t-1}+\mathbf{K}_{t} \boldsymbol{v}_{t} ; \\
\mathbf{P}_{t} & =\left(\mathbf{T}-\mathbf{K}_{t} \mathbf{Z}\right) \mathbf{P}_{t \mid t-1}\left(\mathbf{T}-\mathbf{K}_{t} \mathbf{Z}\right)^{\prime}+\mathbf{R} \mathbf{Q} \mathbf{R}^{\prime}
\end{aligned}
$$

where $\boldsymbol{v}_{t}$ are the model's forecast errors. The remaining vector and matrices have either been defined above or, in the case of $\mathbf{F}_{t}$ and $\mathbf{K}_{t}$, are simply transformations of previously defined matrices. ${ }^{13}$

\subsection{Likelihood function and estimation algorithm}

We are now in a position to write the model's likelihood function as

$$
p\left(\mathbf{y}_{t, t=1}, \ldots, T\right)=\prod_{t=1}^{T}(2 \pi)^{-0.5 n}\left|\mathbf{F}_{t}\right|^{-0.5} \exp \left(-0.5 \boldsymbol{v}_{t}^{\prime} \mathbf{F}_{t}^{-1} \boldsymbol{v}_{t}\right)
$$

where $\boldsymbol{\psi}$ is the vector of model parameters to be estimated. We estimate $\boldsymbol{\psi}$ using the random walk Metropolis-Hastings algorithm (see e.g., Chib and Greenberg (1995)), setting the number of simulations to $S=100,000$ with a burn-in of 50,000. We draw a new realization of $\boldsymbol{\psi}$ according to

$$
\boldsymbol{\psi}_{1}=\boldsymbol{\psi}_{0}+\boldsymbol{\xi}, \boldsymbol{\xi} \sim N(\mathbf{0}, \boldsymbol{\Xi}),
$$

where $\boldsymbol{\Xi}$ is the proposal variance-covariance matrix. A draw $\boldsymbol{\psi}_{1}$ is accepted if

$$
\begin{aligned}
a & \geq u, u \sim U(0,1), \\
a\left(\boldsymbol{\psi}_{1}, \boldsymbol{\psi}_{0}\right) & =\min \left(\frac{p\left(\mathbf{y}_{t, t=1}, \ldots, T \mid \boldsymbol{\psi}_{1}\right) p\left(\boldsymbol{\psi}_{1}\right)}{p\left(\mathbf{y}_{t, t=1}, \ldots, T\right.}, 1\right),
\end{aligned}
$$

\footnotetext{
${ }^{13}$ See Hamilton (1994) or Harvey (1992) for further details regarding the Kalman filter.
} 
where $p(\boldsymbol{\psi})$ is the prior distribution given in Table $1 .{ }^{14}$ The priors regarding the supports for the uniform distributions of the model's parameters reflect non-sample information from: (i) the human capital model derived above; (ii) the empirical literature; (iii) technical considerations regarding the existence of a unique steady-state equilibrium as well as the saddle path stability of the dynamic system; and (iv) empirical considerations regarding the value of long-run human growth in the historical data. To help contextualize the quarterly rates used in Table 1, note that their annual counterparts are as follows: $\gamma=(1.015,1.03), \delta^{k}=(0.0464,0.0514), \delta^{h}=(0.0172,0.0190)$ and $n=(1$, 1.017). The priors for the size of the depreciation rates and the relationship between them, i.e. $\delta^{k}>\delta^{h}$ reflect the findings of Jorgenson and Fraumeni (1989) and Jones et al. (2005).

Table 1: Priors for the parameters, $\boldsymbol{\psi}$

\begin{tabular}{lcl}
\hline \hline & Parameters & Restrictions \\
\hline capital's share & $\alpha$ & $(0.25,0.5)$ \\
discount rate & $\beta$ & $(0.965,0.999)$ \\
$K$ depreciation rate & $\delta^{k}$ & $(0.0114,0.0126)$ \\
$H$ depreciation rate & $\delta^{h}$ & $(0.0043,0.0047)$ \\
utility function param. & $\sigma$ & $(1,3)$ \\
s.d. $A$ shock & $\sigma^{a}$ & $(0,0.05)$ \\
s.d. $B$ shock & $\sigma^{b}$ & $(0,0.05)$ \\
externality parameter & $(1-\theta)$ & $(0,1)$ \\
AR(1) parameter in $A_{t}$ & $\rho^{a}$ & $(0,1)$ \\
AR(1) parameter in $B_{t}$ & $\rho^{b}$ & $(0,1)$ \\
constant term in $A_{t}$ & $A$ & $(0.238,0.263)$ \\
constant term in $B_{t}$ & $B$ & $(0.029,0.032)$ \\
gross rate $N$ growth & $n$ & $(1,1.0042)$ \\
eigenvalues (VAR) & $\lambda_{\{i=1,2,3\}}$ & max $\left|\lambda_{i}\right|<1$ \\
covariances & $\Sigma_{y y}, \Sigma_{c c}, \Sigma_{h h}, \Sigma_{y c}, \Sigma_{y h}, \Sigma_{c h}$ & $\Sigma$ is + semi-definite \\
model solution & unique steady-state exists & exitflag=1 (fsolve.m $)^{*}$ \\
gross rate $H$ growth & $\gamma($ steady-state $)$ & $(1.0037,1.0074)$ \\
\hline
\end{tabular}

* fsolve.m is the Matlab function used to solve the non-linear system of equations comprising the model's steady-state.

\footnotetext{
${ }^{14}$ Note that an extra Metropolis-Hastings step will be required to estimate human capital which is used to de-trend the data. We return to this issue below and will show how the above sampling procedure needs to be modified.
} 


\section{Estimation results}

In this section we start by discussing the data employed and the sampling procedures required to make them stationary. We then present the first two moments of the estimated posterior parameter distributions and numerical standard errors for each parameter. We finally turn to an assessment of the model's ability to explain the observed variation in the measured data, i.e. output, consumption, investment and hours. To this end, we undertake forecast error decompositions (FEDs) which allow us to split the contemporaneous and the $k$-step-ahead forecast error variances of the measured variables into the portions explained by the two technology shocks plus the innovations to the $\operatorname{VAR}(1)$ error system.

\subsection{Data}

To facilitate comparisons with recently estimated RBC models in the literature, we use the U.S. data set employed in Ireland (2004). ${ }^{15}$ These data are quarterly and include per capita output, per capita consumption and hours over the period 1948(1) to 2002(2). Note that output is defined as the sum of consumption and investment and all quantities are in real chained $1992 \$$. The hours data refer to hours of wage and salary workers on private, nonfarm payrolls and the population series is the civilian, non-institutional population, age 16 and over.

\subsubsection{Human capital}

As discussed in section 2.3, the per capita non-stationary quantities in the model need to be normalized by human capital to be rendered stationary. Thus, given the measured data for output, consumption and hours, a starting parameter vector, $\boldsymbol{\psi}^{0}$, and a starting sequence $H_{t}^{0}$ for the human capital stock, the data are de-trended and the parameter vector $\boldsymbol{\psi}$ is estimated concurrently as follows:

1. De-trend the non-stationary data (i.e. output and consumption) by $H_{t}^{0}$.

2. Using $\boldsymbol{\psi}^{0}$ and the stationary data, a realization of the state vector $\boldsymbol{\alpha}_{t}=\left(\begin{array}{lllll}\hat{k}_{t} & \hat{c}_{t} & \hat{e}_{t} & \hat{a}_{t} & \hat{b}_{t}\end{array}\right)^{\prime}, t=0, \ldots, T$ is generated in a multi-move Gibbs sampling step (see, Cater and Kohn (1994) and the Appendix for more details).

\footnotetext{
${ }^{15}$ This data was also used in Malley and Woitek (2009).
} 
3. Recover the deviation of the growth rate of human capital from its steady-state value using

$$
\hat{\gamma}_{t}=\left(\frac{B \theta e^{\theta}}{n \gamma}\right) \hat{e}_{t}+\left(\frac{B e^{\theta}}{n \gamma}\right) \hat{b}_{t}
$$

and calculate the growth rate of human capital from $\gamma_{t}=\hat{\gamma}_{t}+\gamma$, $t=0, \ldots, T$ using the state vector generated in step $2 .{ }^{16}$

4. The starting value $H_{0}^{1}$ is obtained from a random-walk MetropolisHastings step:

$$
H_{0}^{1}=H_{0}^{0}+\phi ; \phi \sim N\left(0, \sigma_{\phi}\right) .
$$

The new sequence $H_{t}^{1}$ is calculated as

$$
H_{t}^{1}=\gamma_{t} H_{t-1}^{1}, \quad t=1, \ldots, T .
$$

$H_{t}^{1}$ replaces $H_{t}^{0}$ if

$$
\begin{aligned}
& a \geq u, \quad u \sim U(0,1),
\end{aligned}
$$

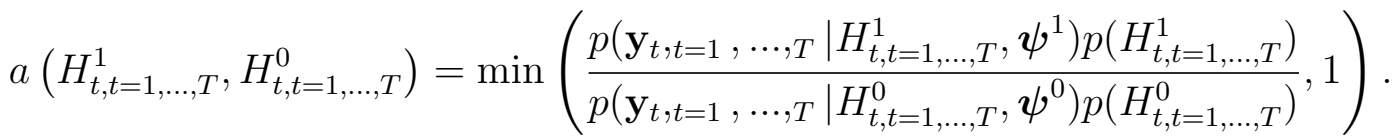

5. The data are next de-trended using $H_{t}^{1}$.

6. Another random-walk Metropolis-Hastings step generates $\boldsymbol{\psi}^{1}$ :

$$
\boldsymbol{\psi}^{1}=\boldsymbol{\psi}^{0}+\boldsymbol{\xi}, \quad \boldsymbol{\xi} \sim N(\mathbf{0}, \boldsymbol{\Xi}) .
$$

The vector $\boldsymbol{\psi}^{1}$ replaces $\boldsymbol{\psi}^{0}$ if

$$
\begin{aligned}
& a \geq u, \quad u \sim U(0,1),
\end{aligned}
$$

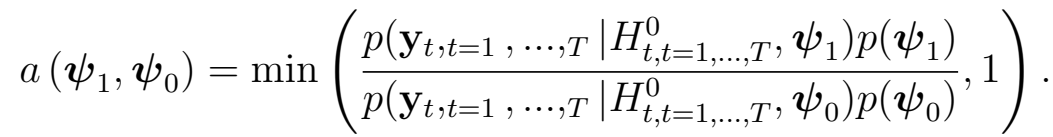

\section{Continue with step 1.}

\footnotetext{
${ }^{16}$ Note the expression for $\widehat{\gamma}_{t}$ can be deduced from the information supplied in the Appendix.
} 


\subsection{Posterior distributions of parameters}

Table 2 summarizes the estimated parameter distributions by reporting their means and standard deviations along with a measure of estimation accuracy based on numerical standard errors, NSE, (see, e.g. Geweke (1992)). ${ }^{17}$

Table 2: Posterior distributions of parameters, $\boldsymbol{\psi}$

\begin{tabular}{lrrr}
\hline \hline & \multicolumn{3}{c}{ Human capital model } \\
& mean & s.d. & $\frac{N S E}{\left|\psi_{j}\right|}$ \\
\hline$\alpha$ & 0.41775 & 0.00341 & 0.00008 \\
$A$ & 0.24634 & 0.00287 & 0.00012 \\
$B$ & 0.03092 & 0.00058 & 0.00019 \\
$\beta$ & 0.97028 & 0.00219 & 0.00002 \\
$\delta_{k}$ & 0.01179 & 0.00027 & 0.00023 \\
$\delta_{h}$ & 0.00449 & 0.00013 & 0.00029 \\
$n$ & 1.00121 & 0.00096 & 0.00001 \\
$\sigma$ & 1.99150 & 0.00470 & 0.00002 \\
$\theta$ & 0.51216 & 0.00280 & 0.00005 \\
$\rho_{a}$ & 0.99425 & 0.00165 & 0.00002 \\
$\rho_{b}$ & 0.99949 & 0.00041 & 0.00000 \\
$\sigma_{a}$ & 0.00500 & 0.00007 & 0.00014 \\
$\sigma_{b}$ & 0.01000 & 0.00000 & 0.00029 \\
$a_{y y}$ & -0.09240 & 0.00251 & 0.00027 \\
$a_{c y}$ & -0.12444 & 0.00245 & 0.00020 \\
$a_{c u}$ & 0.22004 & 0.00425 & 0.00019 \\
$a_{y c}$ & 0.16814 & 0.00401 & 0.00024 \\
$a_{c c}$ & -0.14867 & 0.00184 & 0.00012 \\
$a_{u c}$ & -0.17121 & 0.00474 & 0.00028 \\
$a_{y u}$ & -0.12401 & 0.00183 & 0.00015 \\
$a_{c u}$ & -0.02903 & 0.00048 & 0.00017 \\
$a_{u u}$ & -0.00974 & 0.00040 & 0.00041 \\
$\Sigma_{y y}$ & 0.00113 & 0.00014 & 0.00123 \\
$\Sigma_{c y}$ & 0.00091 & 0.00027 & 0.00303 \\
$\Sigma_{u y}$ & -0.00353 & 0.00019 & 0.00055 \\
$\Sigma_{c c}$ & 0.00391 & 0.00062 & 0.00158 \\
$\Sigma_{c u}$ & -0.00677 & 0.00035 & 0.00052 \\
$\Sigma_{u u}$ & 0.02915 & 0.00093 & 0.00032 \\
\hline & & & \\
\hline
\end{tabular}

\footnotetext{
${ }^{17}$ Note that the ratio reported in the Table 2 is in percent terms, i.e. $\frac{N S E}{\bar{\psi}_{i}} \times 100$. Also note that acceptance rates for the random-walk Metropolis Hastings steps were $65 \%$ for the parameter vector, $30 \%$ for the starting value of the de-trender.
} 
If we start with the means of the posterior parameter distributions containing the largest prior support ranges, it's first interesting to observe that the value of the inter-temporal elasticity of substitution of consumption, $\frac{1}{\sigma}$, is approximately 0.5. This value along with the special case of $\sigma=1$ are often used in a variety of calibration studies. Second, the estimated posterior mean for, $\theta$, suggests that the no externality case, $\theta=1$, is not supported by the data, despite allowing for this possibility in the prior (see Table 1). Third, the size of the autoregressive parameters in the two trend stationary technology processes are highly persistent. ${ }^{18}$ Moreover, the process for the human capital shock is far more persistent than the Hicks-neutral shock, e.g. its half-life is roughly 10 times higher.

If we next consider the remaining parameters not related to the $\operatorname{VAR}(1)$ errors, there are no surprises regarding the sizes of the means given our priors regarding the supports for the various distributions. However, it is interesting to note that the mean value of the variance of the Hicks-neutral technology process, $\sigma_{a}=0.005$, which is consistent with other estimates in the literature, is about half the size of the corresponding value for the human capital technology process, $\sigma_{b}=0.01$.

We finally turn to the spread of these distributions, which allows us to assess the degree of uncertainty associated with size of the means. In general, it appears that all the parameters, both structural and from the VAR(1) model of the errors, are quite concentrated. Moreover, examination of the numerical standard errors as a share of the absolute value of the means of the posteriors reveals that our estimates are generally very precise. Indeed they compare quite favorably with others estimated in the literature.

\subsection{Forecast error decompositions}

Figures 1-4 report the share of the forecast error for output, consumption, investment and hours explained by the two technology shocks (see left axis) and the error system (see right axis). All of the measured data are in terms of stationary log-deviations from the steady-state. The figures contain the decompositions for forecast horizons $k=\{0,1,4,8,12,20,40, \infty\}$ as well as information pertaining to their distributions. ${ }^{19}$ For example, the box plots show the (i) median; (ii) inter-quartile range; and (iii) extreme values at each

\footnotetext{
${ }^{18}$ This echoes the results reported in Ireland (2001) and Malley and Woitek (2009). Also note that the above authors find that the data prefer the trend-stationary to differencestationary technology in the standard RBC model (see the respective papers for further information).

${ }^{19}$ Note that the forecast error decompositions come from the posterior distributions and are based on the draws we keep from each of the chains.
} 
$k$.

The key results to emerge from the forecast error decompositions can be summarized as follows. First, both shocks generally do a better job at explaining the variance of the aggregates under consideration at longer versus shorter forecast horizons. This is mostly explained by the highly persistent nature of both technology shocks. Second, for horizons up to ten years, innovations to $A_{t}$ explain a relatively greater share of the variance of all variables than $B_{t}$ shocks (except for hours). Thus its seems that shocks which simultaneously affect capital and labor instead of labor only dominate at business cycle forecast ranges. Third, at very long horizons the explanatory power of $B_{t}$ is greater than $A_{t}$ shocks (except for investment). The dominance of $B$ shocks in the long run, in addition to the suggestion above, will also explained by the facts that $\rho_{b}>\rho_{a}$ and $\sigma_{b}>\sigma_{a}$. Fourth, and generally consistent with the RBC literature, the human capital model does not do a good job at explaining the variation in hours. ${ }^{20}$ Finally, the uncertainty associated with all of the forecast error decompositions appears to be reasonably small based on the size of the inter-quartile ranges. This is expected given the tightness of the posterior parameter distributions.

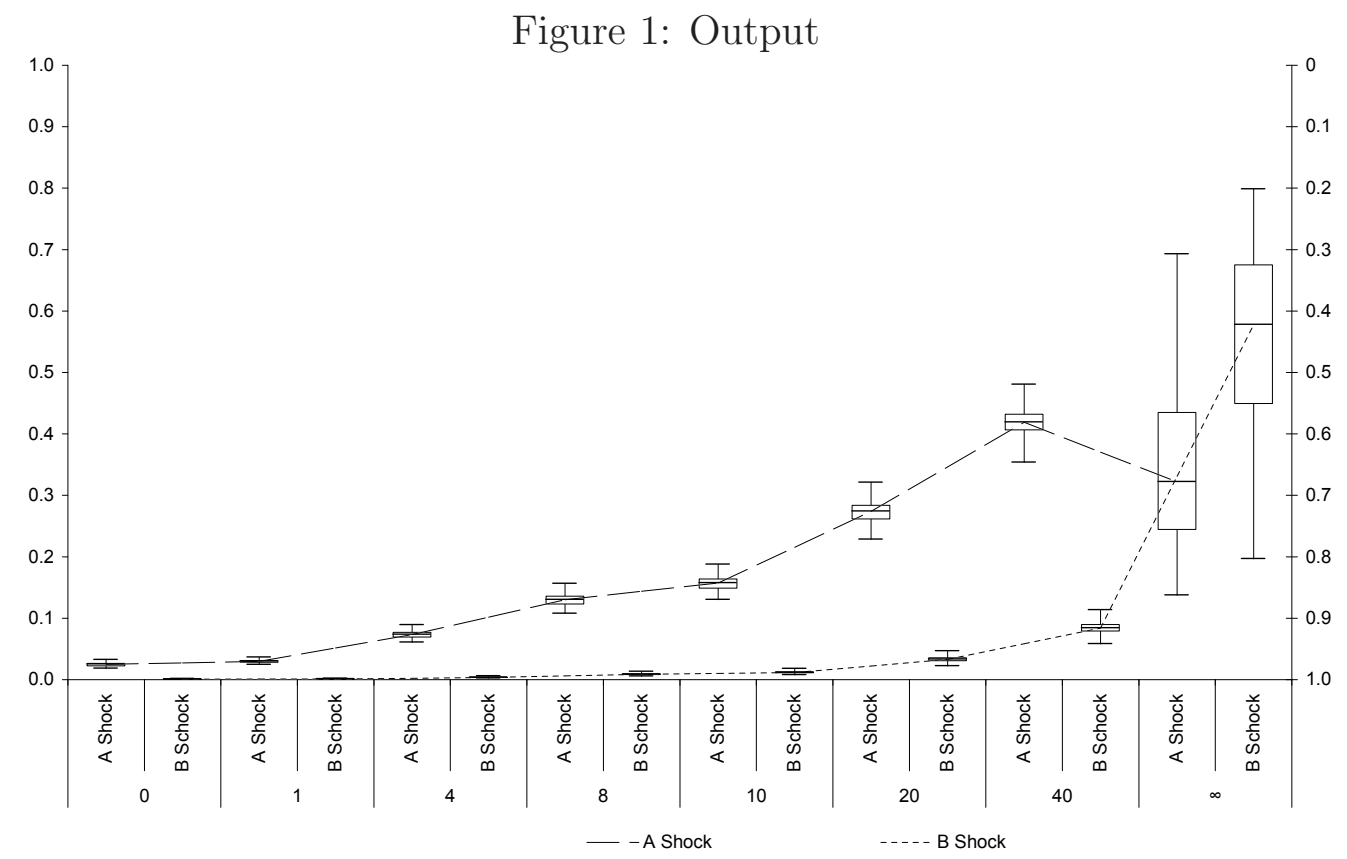

\footnotetext{
${ }^{20}$ We undertake further diagnostics below to try and explain the results for hours and investment in the context of direct comparisons with an estimated RBC model.
} 
Figure 2: Consumption

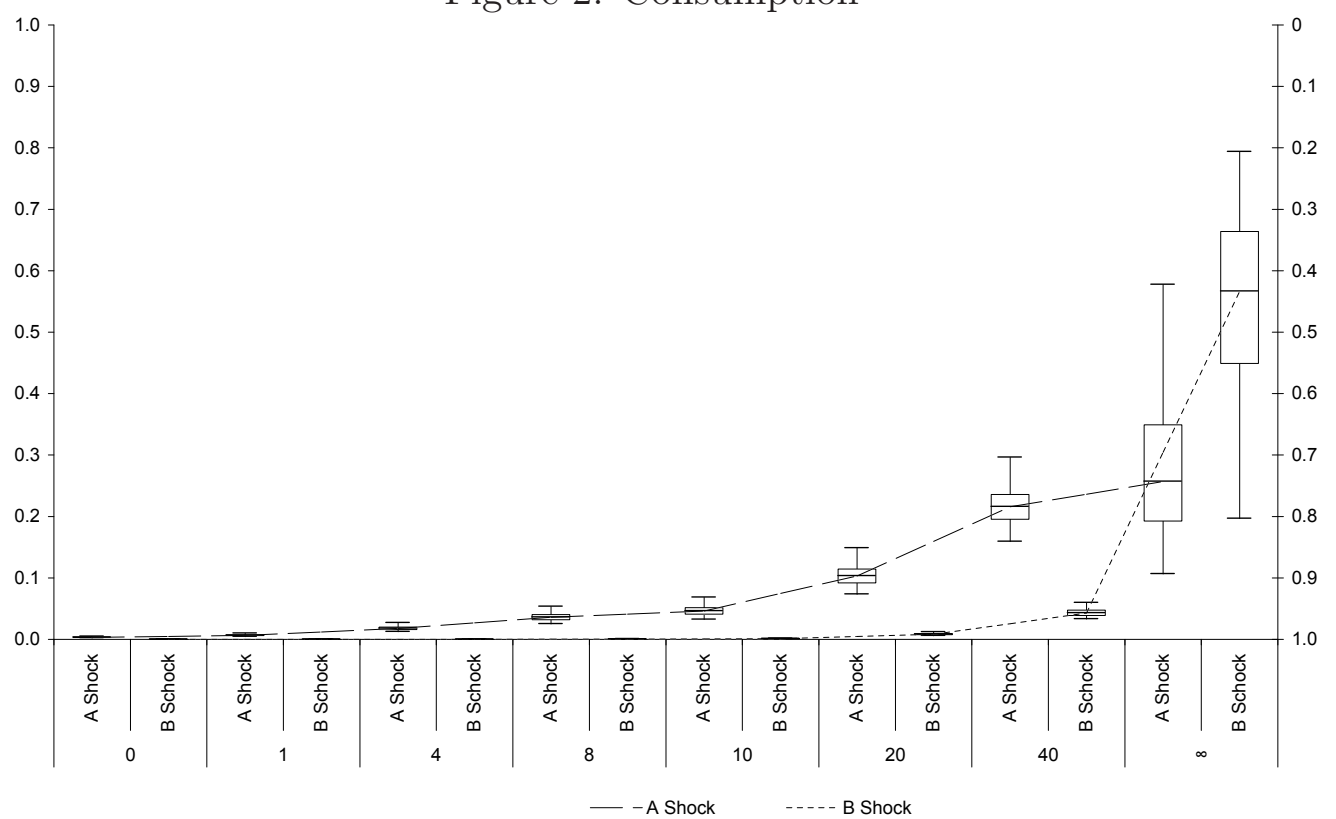

Figure 3: Investment

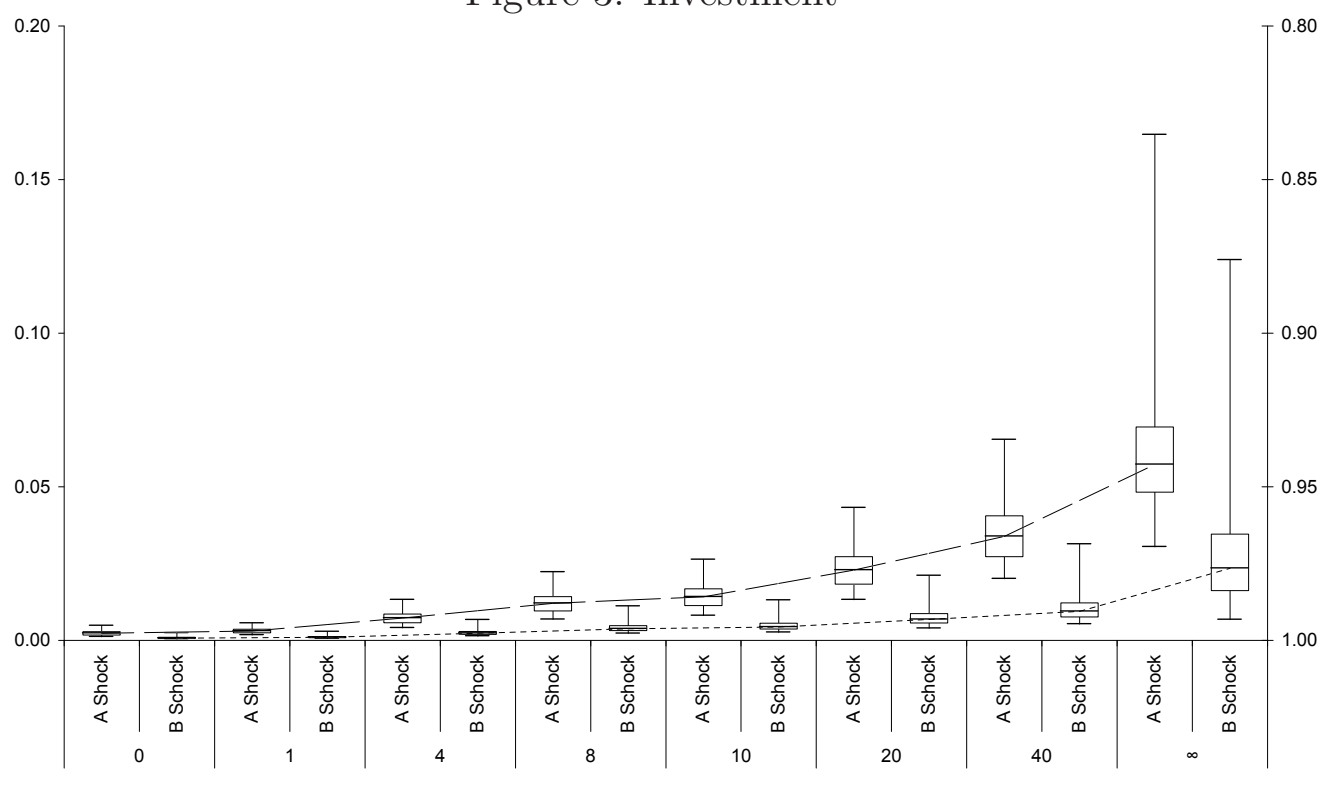

- - A Shock _-.-- B Shock 


\section{Figure 4: Hours}

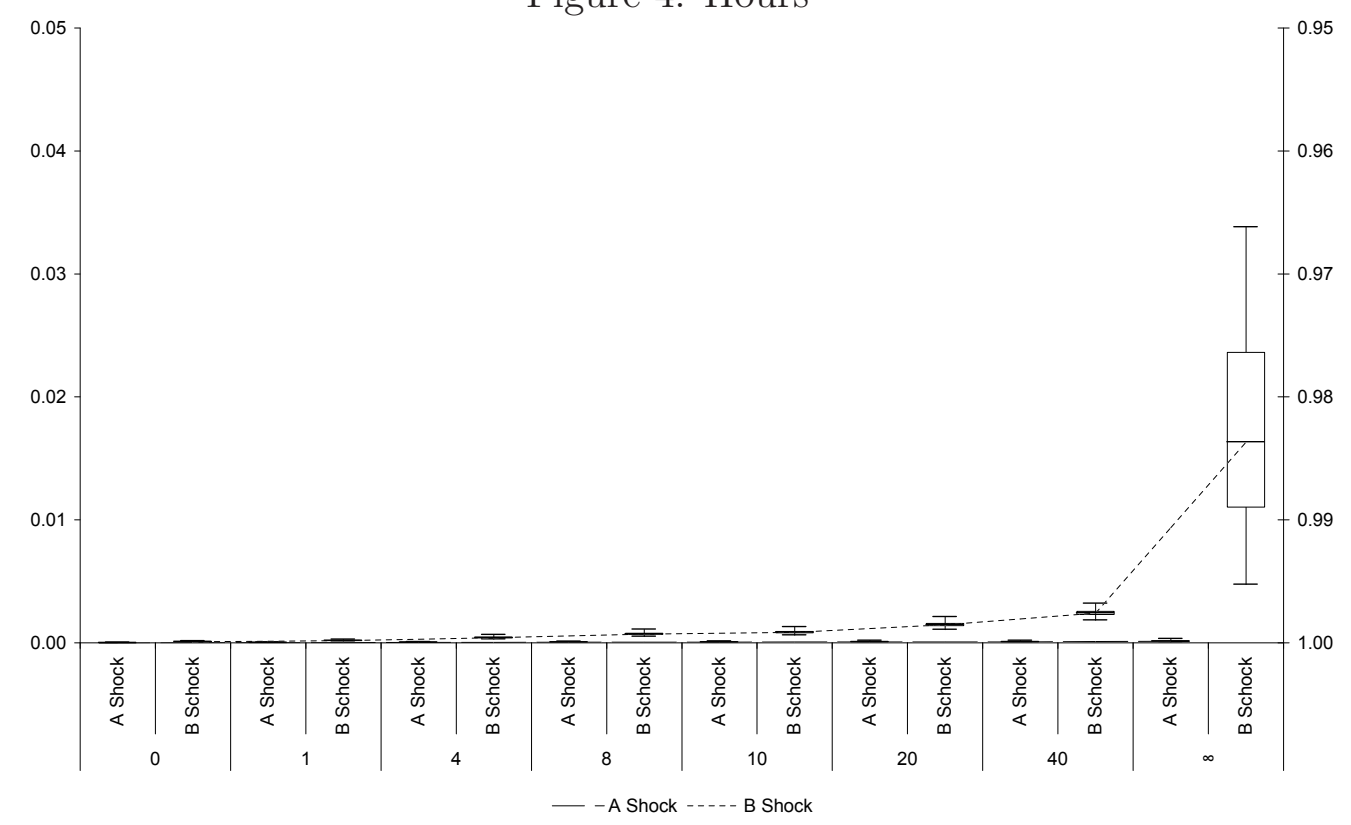

\section{Model comparison}

In this section, we test whether the human capital model or the RBC model are better able to explain the observed variation in output, consumption, investment and hours. We also compare the within sample fit of the competing models using the log-marginal likelihood difference test. To help explain the results of these tests we make use of information obtained from impulse response functions and further forecast error decompositions of the VAR(1) error block. The latter breaks out the relative contributions of each of the errors to explaining the measured data.

\subsection{Estimation results RBC model}

The RBC model that we test against is the industry standard Hansen (1985) model estimated in Ireland (2004) and Malley and Woitek (2009). The main differences between this RBC model and the human capital model estimated above are that endogenous human capital accumulation is replaced by exogenous labor augmenting technical progress and the labor-education choice is replaced by the labor-leisure choice.

To aid the interpretation of the results which follow it is useful to be more specific about the differences between the particular endogenous growth and RBC models used here. For example, to obtain the Hansen (1985) 
model setup using the notation and structure applied above, requires the following changes: (i) re-define the time constraint in eq. (3) to $u_{t}^{h}+l_{t}^{h}=$ 1 , where $l_{t}^{h}$ is the household's leisure time; (ii) re-define the instantaneous utility function in eq. (2) to, $U_{t}=\ln \left(C_{t}^{h}\right)-\mu\left(1-u_{t}^{h}\right)$, where $\mu>0$ is the parameter governing the linearity of work time; (iii) re-define human capital accumulation in eq. (6) to $H_{t+1}^{h}=\eta H_{t}^{h}$, where $\eta>1$ is the gross rate of labor augmenting technical progress and $H_{0}=1$; and finally (iv) set the gross rate of population growth, $n$, defined above eq. (1), to unity. Given these changes, $\mu$ and $\eta$ are the only new structural parameters to appear in following table of posterior distributions of parameters. ${ }^{21}$

For ease of comparison, the estimates from the human capital model in Table 2 have also been included in Table 3. Some of the main differences across models which may help to explain the FEDs and within sample fit tests which follow are: (i) both models have highly persistent $A$ shocks with $A_{R B C}>A_{H C}$; (ii) the inter-temporal elasticity of substitution of consumption is restricted to unity in the RBC case implying $(1 / \sigma)_{R B C}>(1 / \sigma)_{H C}$; (iv) the externality parameter, $(1-\theta)$, is restricted to zero in the RBC case; and $(\mathrm{v})$ the parameter distributions in the human capital model are generally much more accurately estimated than in the RBC model.

To obtain a quantitative impression of how the differences in the parameter estimates in Table 3 affect model outcomes, we next examine the impulse responses of the two models to a temporary 1-standard deviation $A$ shock in Figure 5 below. These are reported as percent differences from the steady-state for 100 quarters.

The plots for the RBC model show the expected results. That is, on impact, increased TFP directly increases output by more than the value of the shock via a rise in the marginal products of capital and labor. These lead agents to work more and to invest and accumulate more capital. There are two competing forces at play with respect to the consumption reaction. The positive one or the income effect arising from the higher returns to working. And the negative one or the substitution effect associated with the fact that $t+1$ returns derived from investing in one unit of physical capital at time $t$ have increased. Figure 5 shows that the income effect dominates. After the initial shock, all variables eventually return to their respective steady-states at a speed which depends on the size of $\rho^{a}$ in the TFP process. Consistent with the estimated values of $\rho^{a}$, Figure 5 also illustrates that TFP is an extremely persistent trend stationary process in both models.

\footnotetext{
${ }^{21}$ See Malley and Woitek (2009, Table 1, p. 11) for the priors used to obtain the estimates for the RBC model reported here.
} 
Table 3: Posterior distributions of parameters, $\boldsymbol{\psi}$

\begin{tabular}{lrrrrrr}
\hline \hline & \multicolumn{2}{c}{ Human capital model } & \multicolumn{3}{c}{ RBC model } \\
$\psi_{j}$ & mean & s.d. & $\frac{N S E}{\left|\psi_{j}\right|}$ & mean & s.d. & $\frac{N S E}{\left|\psi_{j}\right|}$ \\
\hline$\alpha$ & 0.41775 & 0.00341 & 0.00008 & 0.23053 & 0.00005 & 0.00721 \\
$A$ & 0.24634 & 0.00287 & 0.00012 & 5.18544 & 0.00003 & 0.00017 \\
$B$ & 0.03092 & 0.00058 & 0.00019 & na & na & na \\
$\beta$ & 0.97028 & 0.00219 & 0.00002 & 0.99050 & 0.00002 & 0.00069 \\
$\delta_{k}$ & 0.01179 & 0.00027 & 0.00023 & 0.02346 & 0.00003 & 0.04362 \\
$\delta_{h}$ & 0.00449 & 0.00013 & 0.00029 & na & na & na \\
$n$ & 1.00121 & 0.00096 & 0.00001 & na & na & na \\
$\mu$ & na & na & na & 0.00468 & 0.00000 & 0.00744 \\
$\sigma$ & 1.99150 & 0.00470 & 0.00002 & na & na & na \\
$\theta$ & 0.51216 & 0.00280 & 0.00005 & na & na & na \\
$\rho_{a}$ & 0.99425 & 0.00165 & 0.00002 & 0.99825 & 0.00001 & 0.00032 \\
$\rho_{b}$ & 0.99898 & 0.00082 & 0.00001 & na & na & na \\
$\sigma_{a}$ & 0.00500 & 0.00007 & 0.00014 & 0.00540 & 0.00030 & 0.33773 \\
$\sigma_{b}$ & 0.01000 & 0.00014 & 0.00014 & na & na & na \\
$a_{y y}$ & -0.09240 & 0.00251 & 0.00027 & 1.36488 & 0.00003 & 0.00072 \\
$a_{c y}$ & -0.12444 & 0.00245 & 0.00020 & 0.13823 & 0.00003 & 0.00654 \\
$a_{h y}$ & 0.22004 & 0.00425 & 0.00019 & 0.71462 & 0.00004 & 0.00213 \\
$a_{y c}$ & 0.16814 & 0.00401 & 0.00024 & 0.38978 & 0.00002 & 0.00167 \\
$a_{c c}$ & -0.14867 & 0.00184 & 0.00012 & 0.96848 & 0.00005 & 0.00167 \\
$a_{c h}$ & -0.17121 & 0.00474 & 0.00028 & 0.46005 & 0.00002 & 0.00159 \\
$a_{y h}$ & -0.12401 & 0.00183 & 0.00015 & -0.49191 & 0.00003 & 0.00205 \\
$a_{c h}$ & -0.02903 & 0.00048 & 0.00017 & -0.10444 & 0.00003 & 0.00780 \\
$a_{h h}$ & -0.00974 & 0.00040 & 0.00041 & 0.22186 & 0.00002 & 0.00294 \\
$\Sigma_{y y}$ & 0.00113 & 0.00014 & 0.00123 & 0.00008 & 0.00001 & 2.83334 \\
$\Sigma_{c y}$ & 0.00091 & 0.00027 & 0.00303 & 0.00006 & 0.00001 & 2.13174 \\
$\Sigma_{h y}$ & -0.00353 & 0.00019 & 0.00055 & 0.00002 & 0.00001 & 4.31755 \\
$\Sigma_{c c}$ & 0.00391 & 0.00062 & 0.00158 & 0.00004 & 0.00001 & 3.85931 \\
$\Sigma_{c h}$ & -0.00677 & 0.00035 & 0.00052 & 0.00002 & 0.00000 & 3.80877 \\
$\Sigma_{h h}$ & 0.02915 & 0.00093 & 0.00032 & 0.00003 & 0.00001 & 4.32013 \\
\hline & & & & & &
\end{tabular}


Figure 5: Impulse responses
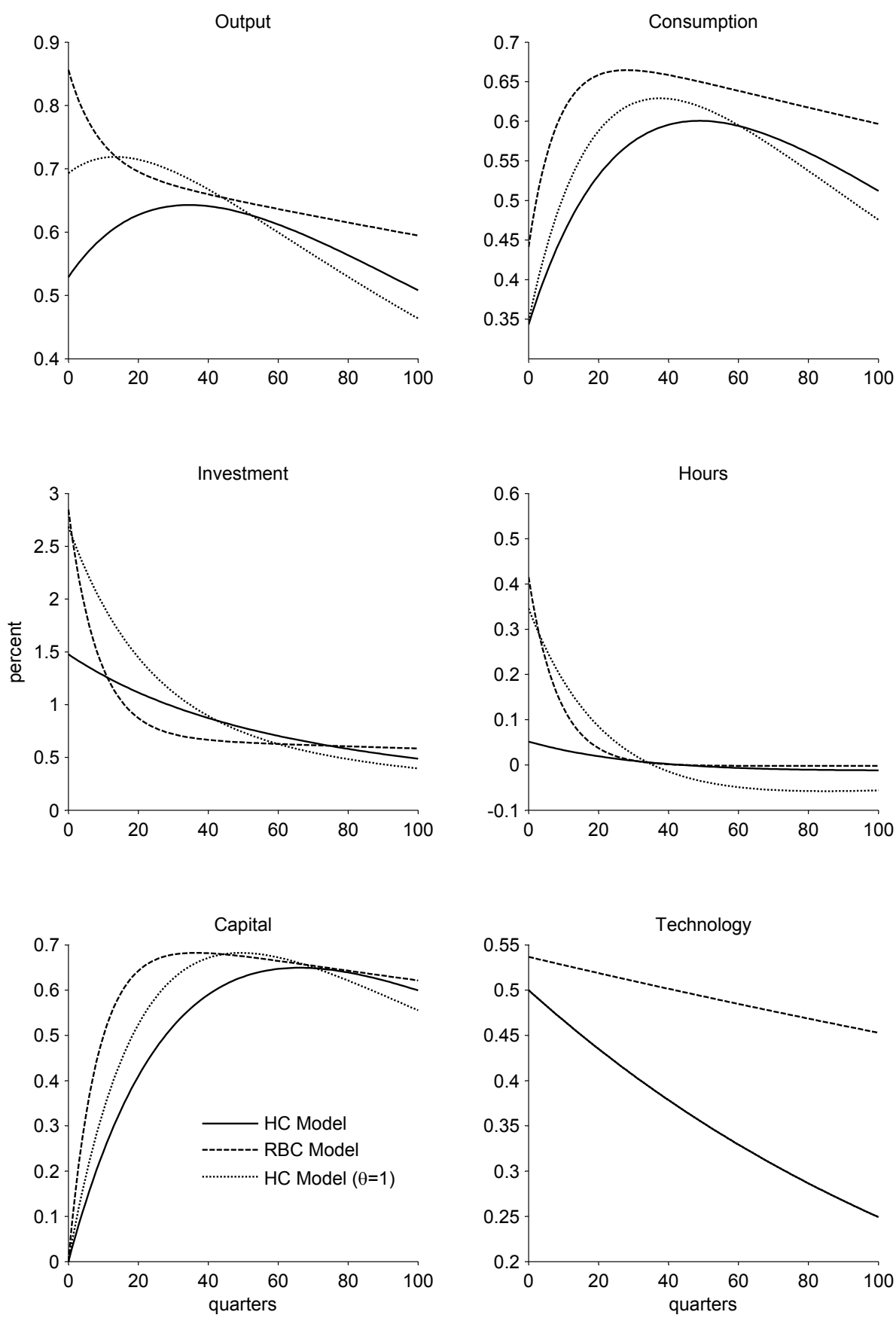
In contrast to the RBC model, besides the fact that the output, consumption and capital responses for the human capital model are relatively lower, the striking difference is that the responses of investment and hours are both much flatter in the human capital model. In an attempt to understand which parameters are driving the differences in the impulse responses across models, we experimented with every common structural parameter including the standard deviation of the shock and found that the externality parameter plays, by far, the most dominant role. In other words, increasing $\theta$ to unity, ceteris paribus, produces impulse responses in the human capital model which are much closer to the RBC case. The results of this exercise, also presented in Figure 5, suggest that in the absence of the human capital externality, more time is devoted to work, $u_{t}$, implying less time to education, $e_{t}$, and hence less human capital growth, $\gamma_{t}$. The latter implies lower human capital accumulation, accordingly all of the per human capital quantities increase, i.e. $\hat{y_{t}}, \hat{c_{t}}, \hat{i_{t}}$ and $\hat{k_{t}}$.

\subsection{Forecast error decompostions: RBC vs human capital model}

With the above quantitative differences between models in mind, we next turn to comparisons of the FEDs in Figures 7-10. The first message from these figures is that the RBC model does much better than the human capital model at the shorter forecast horizons for all variables. However for horizons around 20 quarters to infinity the combined effects of the $A$ and $B$ shocks in the human capital model dominate the $A$ shocks in the RBC model in the case of output. This pattern is repeated for consumption but only in the very long-run. Finally, the combined effect of the two shocks in the human capital model never explains more of the variation in investment and hours than the $A$ shock in the RBC model.

Our impulse response findings from above suggest that the last result can be largely explained by the inelastic response of investment and hours due to the presence of aggregate externalities. To further explore the potential reasons for the differences in the FEDs across models, we decompose the forecast-errors of the model into the proportions explained by innovations to the output, consumption and hours errors below. 
Figure 6: Output

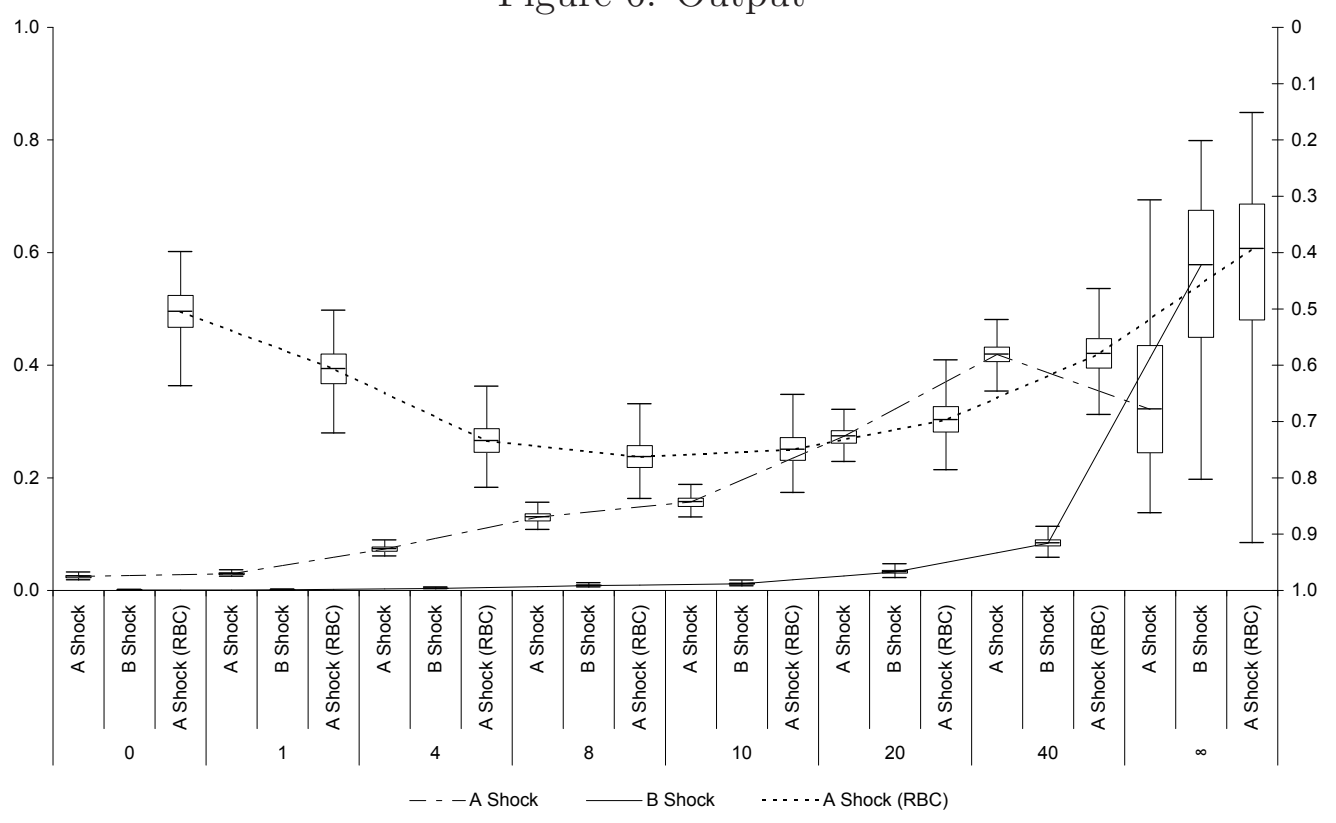

Figure 7: Consumption

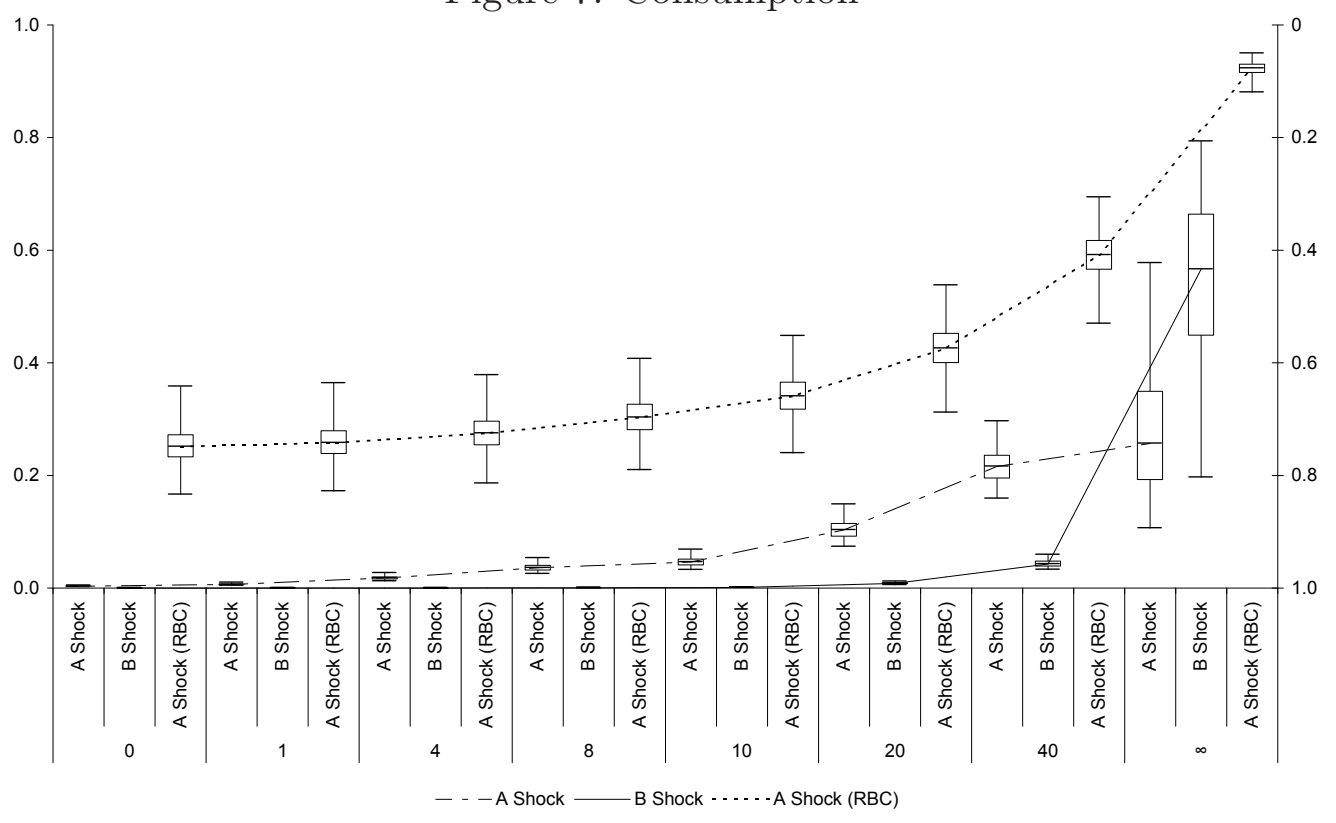


Figure 8: Investment

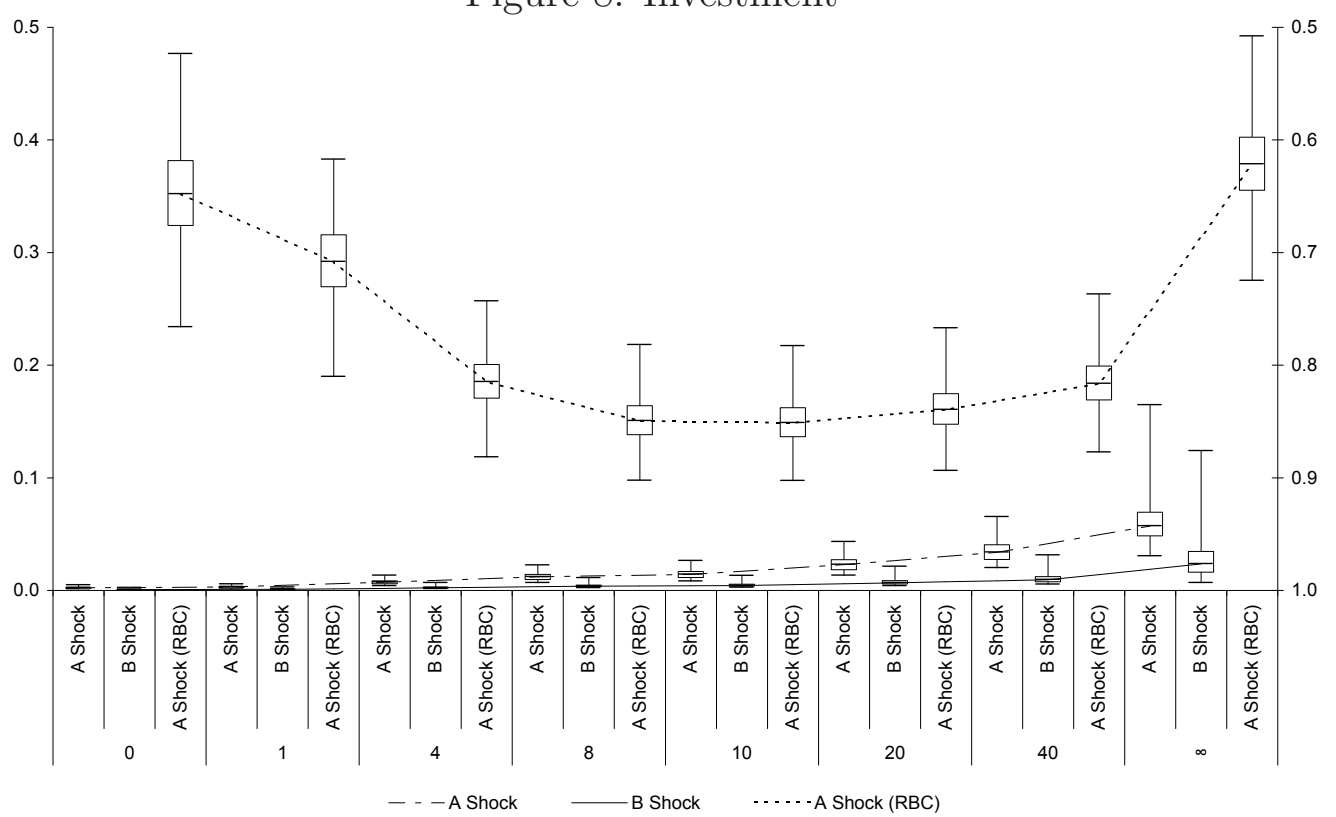

Figure 9: Hours

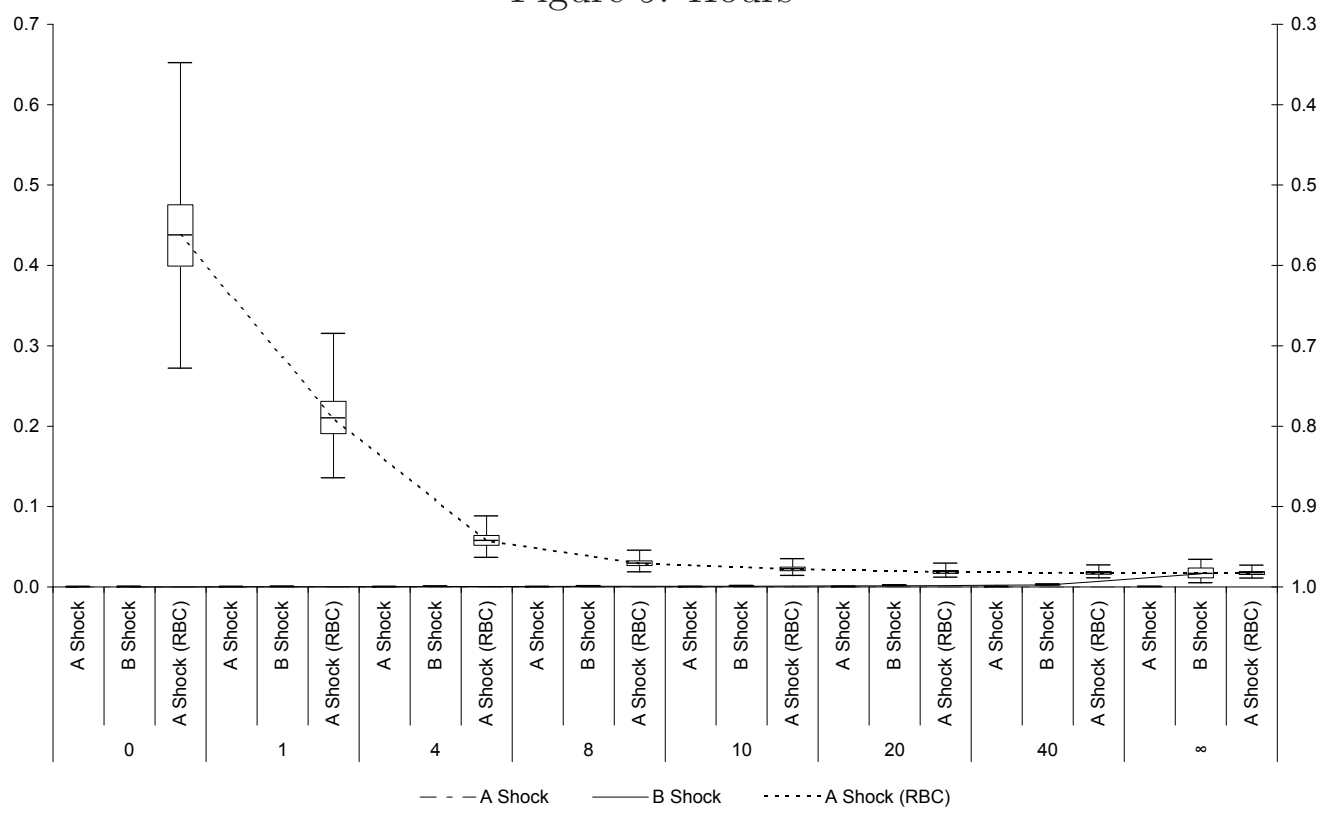




\subsection{Within sample fit}

The forecast error decompositions conducted above suggest that neither of work horse growth models uniformly dominates the other across all variables and forecast horizons. As another means of comparing these models, we next assess their relative abilities to explain the variation in the historical data. To this end we follow Chib and Jeliazkov (2001) and calculate Bayes factors based on marginal likelihoods obtained from the simulated parameter realizations. Let $M_{H C}$ denote the human capital model, and $M_{R B C}$ the RBC model. The Bayes factors for comparing the models are given by the ratio of the two marginal likelihoods for $M_{H C}$ and $M_{R B C}$,

$$
B F_{H C, R B C}=\frac{p\left(\mathbf{y} \mid M_{H C}\right)}{p\left(\mathbf{y} \mid M_{R B C}\right)} .
$$

The marginal likelihood is given by

$$
\begin{aligned}
\ln \hat{p}\left(\mathbf{y} \mid M_{H C}\right)= & \ln p\left(\mathbf{y} \mid \boldsymbol{\psi}_{j}^{\star}, M_{H C}\right)+\ln p\left(\boldsymbol{\psi}^{\star} \mid M_{H C}\right)- \\
& -\ln \hat{p}\left(\boldsymbol{\psi}_{H C, 1}^{\star} \mid \mathbf{y}, M_{H C}\right)-\ln \hat{p}\left(\boldsymbol{\psi}_{H C, 2}^{\star} \mid \mathbf{y}, M_{H C}, \boldsymbol{\psi}_{H C, 1}^{\star}\right),
\end{aligned}
$$

where subscript $j=H C, R B C, \boldsymbol{\psi}_{H C, 1}^{\star}$ is the first parameter block (i.e. the de-trender), and $\boldsymbol{\psi}_{H C, 2}^{\star}$ contains the rest of the parameters. ${ }^{22}$

Based on eqs. (26) and (27) we obtain a value of 847.68 for the logmarginal difference between the human capital and RBC model suggesting that the former provides a far better within sample fit to the data. This result should not be too surprising in light of the numerical standard errors reported in Table 3.

\subsection{Forecast error decompostions of $\operatorname{VAR}(1)$ errors}

\subsubsection{Setup}

To achieve a more detailed understanding of the dynamic interactions between the variables in the $\operatorname{VAR}(1)$ error block and their relationship with the variables in the structural model block, consider the following generic reduced form $\operatorname{VAR}(1)$ model

$$
\mathbf{x}_{t}=\tilde{\mathbf{A}} \mathbf{x}_{t-1}+\tilde{\mathbf{e}}_{t}, \quad \tilde{\mathbf{e}}_{t} \sim N(\mathbf{0}, \tilde{\mathbf{\Sigma}}) .
$$

To calculate impulse responses and forecast error decompositions requires that we solve for $\mathbf{S}$ in the following relation between the structural, $\tilde{\boldsymbol{\epsilon}}_{t}$, and reduced form, $\tilde{\mathbf{e}}_{t}$, errors,

$$
\tilde{\mathbf{e}}_{t}=\mathbf{S} \tilde{\boldsymbol{\epsilon}}_{t}, \quad \tilde{\boldsymbol{\epsilon}}_{t} \sim N(\mathbf{0}, \mathbf{I}) .
$$

\footnotetext{
${ }^{22}$ See the Appendix for the derivation of eq. (27).
} 
Technically, any square root of $\tilde{\boldsymbol{\Sigma}}$ can be used since $\tilde{\boldsymbol{\Sigma}}=\mathbf{S S}^{\prime}$.

Given that we have not restricted the $\operatorname{VAR}(1)$ coefficient matrix A nor the VAR(1) co-variance matrix $\boldsymbol{\Sigma}$ matrix in eqs. (18-20) to be diagonal, the measurement/specification error block can be seen as a reduced form VAR(1) as in eq. (28). In contrast, the structure of the lower $(2 \times 2)$ block of the matrix $\tilde{\mathbf{T}}$ in eq. (19) and the variance-covariance matrix $\mathbf{Q}$ in eq. (20) ensures that the structural shocks, $\boldsymbol{\epsilon}_{t}=\left[\begin{array}{ll}\epsilon_{t}^{a} & \epsilon_{t}^{b}\end{array}\right]^{\prime}$, are identified. Thus, to calculate the FEDs for our VAR(1) system we use a principal square root of $\mathbf{Q}$ (which, recall, also incorporates $\Sigma$ ).

\subsubsection{Results}

The FEDs for the VAR(1) errors of the human capital and RBC models are presented in the last three columns of Tables 4 and 5 respectively. ${ }^{23}$ The first two columns of Table 4 and the first column of Table 5 correspond with the median values for the two technology shocks presented in Figures 6-9.

If we first concentrate on the variables not well explained by shocks to productivity in Table 4 (i.e. investment and hours) it appears that innovations to the consumption error, $\nu^{c}$, are the most important in explaining fluctuations in investment across all forecast horizons. These are as high as 69 percent contemporaneously and fall to 53 percent at infinity. Second, own innovations to the hours error, $\nu^{u}$, explain the vast preponderance of the variation in hours for all forecast horizons. For example these proportions never fall below 94 percent.

In contrast, Table 5 for the RBC model shows that the variation in investment is explained by shocks to output, $\nu^{y}$, instead of shocks to $\nu^{c}$. These proportions follow a hump-shaped pattern starting at 49 percent contemporaneously, peaking at 73 percent at 4 quarters and falling to 47 percent at infinity. Also in contrast to the human capital model, except for the contemporaneous period, own shocks to hours never dominate the remaining shocks. Instead the explanatory power rests with both output and consumption error shocks where former dominates the latter across all forecast horizons. The FEDs for the $\nu^{y}$ shock start at 40 percent in quarter 1 , peak at 55 percent in quarter 4 and fall to 49 percent at infinity. Whereas the FEDs for the $\nu^{c}$ shock increase monotonically, starting at 22 percent in quarter 1 and peaking at 48 percent at infinity.

Thus the FEDs for the $\operatorname{VAR}(1)$ errors generally suggest that the explanatory power of the economic block in either model might be improved

\footnotetext{
${ }^{23}$ To save space we have not presented details pertaining to the distributions of the FEDs for the error block, which are at least as accurate as the FEDs presented above. These will make them available upon request.
} 
by adding varying degrees of cyclical structure to output, consumption and hours. In particular, it appears that the human capital model might most benefit from extra structure for consumption and work/education time. ${ }^{24}$ Whereas the RBC model's ability to better capture the dynamics in the data may be increased by adding more structure to consumption and output.

One way to achieve the above would be to ensure that the models' relevant equilibrium conditions take the form of second-order difference equations. ${ }^{25}$ Incorporating these allows for the possibility of cycles if complex conjugate roots are present. In the context of the roots of the characteristic equation, $\lambda_{i}=0.5\left[a \pm \sqrt{a^{2}+4 b}\right]$, of a generic second-order difference equation, $z_{t+1}=$ $a z_{t}+b z_{t-1}$, the necessary condition for the presence of cycles is that $a^{2}+4 b<$ 0 which implies that $b<0$.

Including higher-order equilibrium conditions, without fundamentally altering the structure of the models under consideration, is straightforward. For example, internal consumption habits, capital adjustment costs and labor/education adjustment costs could be added to the structural model. Anyone of these or some combination, depending on their data consistency and whether the condition for cycles is met, would likely have the desired effect of shifting explanatory power from the $\operatorname{VAR}(1)$ error block back to the model.

In contrast, if redistributing cyclicality back to the model block is not the objective, a popular alternative approach is to impose identifying restrictions on the VAR(1) errors at the estimation stage by assuming for example that the $\operatorname{VAR}(1)$ coefficient matrix $\mathbf{A}$ and its corresponding co-variance matrix $\boldsymbol{\Sigma}$ matrix in eqs. (18-20) are diagonal. ${ }^{26}$ This in turn would give a structural interpretation to the components of the VAR(1) errors. For example, innovations to consumption, output and hours could then be identified as taste, demand and preference shocks respectively. The marginal likelihood tests, as conducted above, could then be applied as means of allowing the data to discriminate between the different approaches.

\footnotetext{
${ }^{24}$ Given that work time follows residually from education time, the FEDs for each are identical.

${ }^{25}$ While first-order difference equations with negative roots can generate cycles with a period of 2 time-units, these are generally not a good description of the type of fluctuations present in aggregate macroeconomic data.

${ }^{26}$ Dependent on the nature of the shocks, there are a variety of other possible identification schemes which could be employed. For example the Cholesky decomposition of $\boldsymbol{\Sigma}$ would be sufficient even when the $\mathbf{A}$ matrix is non-diagonal.
} 
Table 4: HC Model - forecast error decompositions of VAR(1) errors

\begin{tabular}{|c|c|c|c|c|c|c|}
\hline \multirow[b]{2}{*}{ Quarters } & \multirow[b]{2}{*}{ Variable } & \multicolumn{5}{|c|}{ Shocks } \\
\hline & & $a$ & $b$ & $\nu^{y}$ & $\nu^{c}$ & $\nu^{u}$ \\
\hline \multirow{4}{*}{0} & $y$ & 0.0250 & 0.0008 & 0.6840 & 0.0295 & 0.2694 \\
\hline & $c$ & 0.0032 & 0.0001 & 0.0106 & 0.7408 & 0.2368 \\
\hline & $i$ & 0.0023 & 0.0007 & 0.2494 & 0.6897 & 0.0329 \\
\hline & $u$ & 0.0000 & 0.0001 & 0.0102 & 0.0318 & 0.9578 \\
\hline \multirow{4}{*}{1} & $y$ & 0.0297 & 0.0010 & 0.4052 & 0.1019 & 0.4689 \\
\hline & $c$ & 0.0065 & 0.0001 & 0.0151 & 0.7378 & 0.2311 \\
\hline & $i$ & 0.0031 & 0.0010 & 0.1719 & 0.5724 & 0.2301 \\
\hline & $u$ & 0.0000 & 0.0002 & 0.0113 & 0.0338 & 0.9543 \\
\hline \multirow{4}{*}{4} & $y$ & 0.0736 & 0.0035 & 0.3839 & 0.0978 & 0.4466 \\
\hline & $c$ & 0.0179 & 0.0001 & 0.0149 & 0.7276 & 0.2300 \\
\hline & $i$ & 0.0072 & 0.0023 & 0.1714 & 0.5684 & 0.2291 \\
\hline & $u$ & 0.0000 & 0.0004 & 0.0113 & 0.0344 & 0.9535 \\
\hline \multirow{4}{*}{8} & $y$ & 0.1302 & 0.0086 & 0.3580 & 0.0914 & 0.4166 \\
\hline & $c$ & 0.0360 & 0.0005 & 0.0146 & 0.7140 & 0.2259 \\
\hline & $i$ & 0.0120 & 0.0038 & 0.1702 & 0.5649 & 0.2277 \\
\hline & $u$ & 0.0001 & 0.0007 & 0.0113 & 0.0344 & 0.9532 \\
\hline \multirow{4}{*}{10} & $y$ & 0.1573 & 0.0118 & 0.3452 & 0.0884 & 0.4018 \\
\hline & $c$ & 0.0462 & 0.0011 & 0.0144 & 0.7060 & 0.2233 \\
\hline & $i$ & 0.0142 & 0.0044 & 0.1697 & 0.5632 & 0.2271 \\
\hline & $u$ & 0.0001 & 0.0009 & 0.0113 & 0.0344 & 0.9530 \\
\hline \multirow{4}{*}{20} & $y$ & 0.2739 & 0.0329 & 0.2867 & 0.0742 & 0.3335 \\
\hline & $c$ & 0.1032 & 0.0083 & 0.0132 & 0.6574 & 0.2085 \\
\hline & $i$ & 0.0229 & 0.0068 & 0.1677 & 0.5562 & 0.2244 \\
\hline & $u$ & 0.0001 & 0.0015 & 0.0113 & 0.0344 & 0.9525 \\
\hline \multirow{4}{*}{40} & $y$ & 0.4191 & 0.0841 & 0.2046 & 0.0532 & 0.2363 \\
\hline & $c$ & 0.2159 & 0.0428 & 0.0105 & 0.5512 & 0.1744 \\
\hline & $i$ & 0.0338 & 0.0094 & 0.1651 & 0.5481 & 0.2210 \\
\hline & $u$ & 0.0001 & 0.0024 & 0.0112 & 0.0343 & 0.9516 \\
\hline \multirow{4}{*}{$\infty$} & $y$ & 0.3218 & 0.5779 & 0.0380 & 0.0095 & 0.0441 \\
\hline & $c$ & 0.2567 & 0.5664 & 0.0016 & 0.1221 & 0.0380 \\
\hline & $i$ & 0.0573 & 0.0235 & 0.1564 & 0.5286 & 0.2120 \\
\hline & $u$ & 0.0001 & 0.0163 & 0.0111 & 0.0338 & 0.9382 \\
\hline
\end{tabular}


Table 5: RBC Model - forecast error decompositions

\begin{tabular}{|c|c|c|c|c|c|}
\hline \multirow[b]{2}{*}{ Quarters } & \multirow[b]{2}{*}{ Variables } & \multicolumn{4}{|c|}{ Shocks } \\
\hline & & $a$ & $\nu^{y}$ & $\nu^{c}$ & $\nu^{u}$ \\
\hline \multirow{4}{*}{0} & $y$ & 0.4951 & 0.4226 & 0.0442 & 0.0357 \\
\hline & $c$ & 0.2513 & 0.0860 & 0.6227 & 0.0366 \\
\hline & $i$ & 0.3520 & 0.4854 & 0.1447 & 0.0121 \\
\hline & $u$ & 0.4375 & 0.1339 & 0.0712 & 0.3535 \\
\hline \multirow{4}{*}{1} & $y$ & 0.3932 & 0.4825 & 0.0967 & 0.0251 \\
\hline & $c$ & 0.2579 & 0.1113 & 0.5951 & 0.0327 \\
\hline & $i$ & 0.2920 & 0.6291 & 0.0660 & 0.0084 \\
\hline & $u$ & 0.2098 & 0.4019 & 0.2168 & 0.1668 \\
\hline \multirow{4}{*}{4} & $y$ & 0.2654 & 0.5197 & 0.1994 & 0.0124 \\
\hline & $c$ & 0.2748 & 0.1696 & 0.5298 & 0.0240 \\
\hline & $i$ & 0.1852 & 0.7295 & 0.0798 & 0.0039 \\
\hline & $u$ & 0.0575 & 0.5527 & 0.3437 & 0.0445 \\
\hline \multirow{4}{*}{8} & $y$ & 0.2369 & 0.4912 & 0.2598 & 0.0082 \\
\hline & $c$ & 0.3033 & 0.2039 & 0.4734 & 0.0177 \\
\hline & $i$ & 0.1506 & 0.7018 & 0.1433 & 0.0027 \\
\hline & $u$ & 0.0292 & 0.5456 & 0.4008 & 0.0229 \\
\hline \multirow{4}{*}{10} & $y$ & 0.2411 & 0.4735 & 0.2743 & 0.0075 \\
\hline & $c$ & 0.3211 & 0.2086 & 0.4526 & 0.0158 \\
\hline & $i$ & 0.1480 & 0.6854 & 0.1623 & 0.0026 \\
\hline & $u$ & 0.0248 & 0.5350 & 0.4193 & 0.0197 \\
\hline \multirow{4}{*}{20} & $y$ & 0.3027 & 0.4041 & 0.2835 & 0.0064 \\
\hline & $c$ & 0.4258 & 0.1892 & 0.3721 & 0.0112 \\
\hline & $i$ & 0.1606 & 0.6442 & 0.1903 & 0.0025 \\
\hline & $u$ & 0.0186 & 0.5004 & 0.4637 & 0.0160 \\
\hline \multirow{4}{*}{40} & $y$ & 0.4203 & 0.3293 & 0.2429 & 0.0054 \\
\hline & $c$ & 0.5916 & 0.1358 & 0.2641 & 0.0076 \\
\hline & $i$ & 0.1837 & 0.6215 & 0.1897 & 0.0025 \\
\hline & $u$ & 0.0172 & 0.4887 & 0.4772 & 0.0156 \\
\hline \multirow{4}{*}{$\infty$} & $y$ & 0.6036 & 0.2246 & 0.1668 & 0.0037 \\
\hline & $c$ & 0.9232 & 0.0255 & 0.0496 & 0.0014 \\
\hline & $i$ & 0.3790 & 0.4722 & 0.1447 & 0.0019 \\
\hline & $u$ & 0.0171 & 0.4877 & 0.4783 & 0.0156 \\
\hline
\end{tabular}




\section{Conclusions}

This paper has attempted to contribute to the on-going empirical debate regarding the role of technology shocks in explaining aggregate fluctuations in the postwar U.S. data. To this end we have employed a sectoral decomposition of productivity shocks using a standard endogenous growth human capital setup with external effects and Bayesian estimation methods. We have also compared the results from the human capital model with a similarly estimated RBC model.

These have led to the following key findings: (i) in the two-sector model, TFP shocks explain a greater share of output and consumption variation at shorter-forecast horizons whereas human capital productivity innovations dominate at longer ones; (ii) the combined explanatory power of the two technology shocks in the human capital model is greater than the Hicksneutral shock in the RBC model in the medium- and long-term for output and consumption; (iii) the two-sector model provides a significantly better overall fit to the historical data; however (iv) the RBC model outperforms the human capital model with respect to explaining the observed variation in investment and hours.

Application of the hybrid approach to estimation has also suggested that, short of a fundamental re-specification of the model setup, the explanatory power of the two workhorse growth models might be improved by adding more cyclical structure to determinants of consumption and work/education time in the human capital model and consumption and output in the RBC model. In future work we intend to pursue these extensions and to test these against alternatives which rely on imposing identifying restrictions on the VAR component of the model. 


\section{References}

[1] Aghion, P. and P. Howitt (1997). Endogenous Growth Theory. Cambridge, MA. MIT Press.

[2] Azariadis C. and A. Drazen (1990). Threshold externalities in economic development, Quarterly Journal of Economics, 105, 501-526.

[3] Barro, R. and X. Sala-i-Martin (2004). Economic Growth, MIT Press.

[4] Barro R. (2001). Human capital and growth, American Economic Review, $91,12-17$.

[5] Barro R. (1991). Economic growth in a cross section of countries, Quarterly Journal of Economics, 106, 407-43.

[6] Benhabib J. and M. Spiegel (1994). The role of human capital in economic development: Evidence from aggregate cross-country data, Journal of Monetary Economics, 34, 143-73.

[7] Bils M. and P. Klenow (2000). Does schooling cause growth?, American Economic Review, 90, 1160-1183.

[8] Carter, C. and R. Kohn (1994). On Gibbs sampling for state space models, Biometrika, 81, 541-553.

[9] Chib, S. and I. Jeliatzkov (2001). Marginal likelihood from the Metropolis-Hastings output, Journal of the American Statistical Association, 96, 270-281.

[10] Chib, S. and Greenberg, E. (1995). Understanding the MetropolisHastings algorithm, American Statistician, 49, 327-335.

[11] de la Fuente, A. and R. Domenech (2006). Human capital in growth regressions: how much difference does data quality make?, Journal of the European Economic Association, 4, 1-36.

[12] DeJong, D., Ingram, B. and C. Whiteman (2000a). Keynesian impulses versus Solow residuals: identifying sources of business cycle fluctuations, Journal of Applied Econometrics, 15, 311-329.

[13] DeJong, D., Ingram, B. and C. Whiteman (2000b). A Bayesian approach to dynamic macroeconomics, Journal of Econometrics, 98, 203-223.

[14] DeJong, D. and B. Ingram (2001). The cyclical behavior of skill acquisition, Review of Economic Dynamics 4, 536-561 
[15] Fernndez-Villaverde, J. and J. Rubio-Ramírez (2005), Estimating dynamic equilibrium economies: linear versus nonlinear likelihood, Journal of Applied Econometrics, 20, 891-910.

[16] Geweke J. (1992). Evaluating the accuracy of sampling-based approaches to the calculation of posterior moments. In: Bernardo, J.M., Berger, J.O., Dawid, A.P., Smith, A.F.M. (Eds.), Bayesian Statistics, Vol. 4. Oxford University Press, Oxford, 169-193.

[17] Hamilton, J. (1994). Time Series Analysis, Princeton University Press, Princeton, New Jersey.

[18] Harvey, A. (1992). Forecasting, Structural Time Series Models and the Kalman Filter, Cambridge University Press.

[19] Ireland, P. (2008). Productivity and US macroeconomic performance: Interpreting the past and predicting the future with a two-sector real business cycle model, Review of Economic Dynamics, 11, 473-492.

[20] Ireland, P. (2004). A method for taking models to the data, Journal of Economic Dynamics and Control, 24, 1205-1226.

[21] Ireland, P. (2001). Technology shocks and the business cycle: an empirical investigation, Journal of Economic Dynamics and Control, 25, 703-719.

[22] Jones, L., Manuelli, R. and H. Siu (2005). Fluctuations in convex models of endogenous growth, II: Business cycle properties, Review of Economic Dynamics, 8, 805-828.

[23] Jorgenson D. and B. Fraumeni (1989). The accumulation of human and nonhuman capital, 1948-1984, in R.E. Lipsey and H.S. Tice (eds.), The Measurement of Saving, Investment and Wealth, Studies in Income and Wealth, Vol. 52, Chicago, University of Chicago Press.

[24] Kim, D. and C. Lee (2007). On-the-job human capital accumulation in a real business cycle model: Implications for intertemporal substitution elasticity and labor hoarding, Review of Economic Dynamics, 10, 494518.

[25] King, R. and S. Rebelo (1999). Resuscitating real business cycles, in: J. B. Taylor \& M. Woodford (ed.), Handbook of Macroeconomics, Vol. 1, 927-1007, Elsevier. 
[26] Klein, P. (2000). Using the generalized Schur form to solve a multivariate linear rational expectations model, Journal of Economic Dynamics and Control, 24, 1405-1423.

[27] Klenow, P. and A. Rodríguez-Clare (2005). Externalities and Growth, Handbook of Economic Growth, Vol. 1A, P. Aghion and S. Durlauf, eds., 817-861.

[28] Kydland, F. and E. Prescott (1982). Time to build and aggregate fluctuations, Econometrica, 50, 1345-1371.

[29] Lucas R. (2002). Lectures on Economic Growth, Harvard University Press.

[30] Lucas R. (1988). On the mechanics of economic development, Journal of Monetary Economics, 22, 3-42.

[31] Malley, J. and U. Woitek (2009). Technology shocks and aggregate fluctuations in an estimated hybrid RBC model, CESifo Working Paper, 2626 .

[32] Mankiw, G., Romer. P. and D. Weil (1992). A Contribution to the empirics of economic growth, Quarterly Journal of Economics, 107, 407-437.

[33] Perli, R. and P. Sakellaris (1998). Human capital formation and business cycle persistence, Journal of Monetary Economics, 42, 67-92.

[34] Pesaran, M. and Y. Shin (1998). Generalized impulse response analysis in linear multivariate models, Economics Letters, 58, 17-29.

[35] Rebelo, S. (2005). Real business cycle models: past, present and future, Scandinavian Journal of Economics, 107, 217-238.

[36] Romer P. (1986). Increasing returns and long-run growth, Journal of Political Economy, 94, 1002-1037.

[37] Tamura R. (1991). Income convergence in an endogenous growth model, Journal of Political Economy, 99, 522-540.

[38] Temple J. (2001). Generalizations that aren't? Evidence on education and growth, European Economic Review, 45, 905-918. 


\section{Appendix:}

\subsection{First-order approximation}

To solve the model, we take the first-order Taylor series expansion of the non-linear stationary DCE in eq. (15) and the exogenous processes in eq. (16) around the steady-state. After substituting out the Lagrange multipliers and the growth of human capital, the linearized system which we solve to obtain the state space form in eq. (17) is:

$$
\begin{aligned}
&-\hat{y}_{t}-\omega_{4} \hat{e}_{t}+\omega_{5} \hat{k}_{t}+\hat{a}_{t}=0 \\
& \omega_{2} \hat{c}_{t}-\hat{y}_{t}+\omega_{1}\left(\omega_{6} \hat{e}_{t}+\omega_{7} \hat{b}_{t}\right)-\omega_{3} \hat{k}_{t}=-\omega_{1} \hat{k}_{t+1} \\
&-\omega_{8} \hat{c}_{t}+\omega_{8}\left(\omega_{6} \hat{e}_{t}+\omega_{7} \hat{b}_{t}\right)=\omega_{9} E_{t} \hat{y}_{t+1}-\omega_{8} E_{t} \hat{c}_{t+1}-\omega_{9} E \hat{k}_{t+1} \\
&-\omega_{8} \hat{c}_{t}+\hat{y}_{t}+\omega_{23} \hat{e}_{t}+\omega_{24} \hat{b}_{t}=-\omega_{19} E_{t} \hat{c}_{t+1}+\omega_{20} E_{t} \hat{y}_{t+1} \\
& \\
& \rho^{a} \hat{a}_{t}=\omega_{21} E_{t} \hat{b}_{t+1}+\omega_{22} E_{t} \hat{e}_{t+1} \\
& \rho^{b} \hat{b}_{t}=\hat{b}_{t+1}-\varepsilon_{t+1}^{b}
\end{aligned}
$$

where for any variable $x_{t}, \hat{x}_{t}=\ln \left(x_{t} / x\right) ; x$ is the model-consistent steadystate value of $x_{t} ; \hat{y}_{t}$ is the control variable; $\hat{c}_{t}$ and $\hat{u}_{t}$ are jump variables; $\hat{k}_{t}$ is the state variable; $\hat{a}_{t}$ and $\hat{b}_{t}$ are the two exogenous processes; and the $\omega_{i}$ coefficients are defined in the following table.

\begin{tabular}{lll}
\hline \multicolumn{3}{c}{ Appendix Table 1: Parameter convolutions } \\
\hline \hline$\omega_{1} \equiv \frac{n \gamma k}{y}$ & $\omega_{9} \equiv \frac{\alpha y}{\beta^{-1} \gamma^{\sigma} k}$ & $\omega_{17} \equiv \omega_{8}$ \\
$\omega_{2} \equiv \frac{c}{y}$ & $\omega_{10} \equiv \frac{e}{1-e}-\theta+1$ & $\omega_{18} \equiv \frac{\omega_{11}}{\omega_{11}+\omega_{12}}$ \\
$\omega_{3} \equiv \frac{\left(1-\delta^{k}\right) k}{y}$ & $\omega_{11} \equiv c^{-\sigma}(1-\alpha) y$ & $\omega_{19}=\omega_{8} \omega_{13}+\omega_{16}$ \\
$\omega_{4} \equiv \frac{(1-\alpha) e}{1-e}$ & $\omega_{12}=\lambda^{b}\left(1-\delta^{h}\right)+B \theta e^{\theta}$ & $\omega_{20}=\omega_{13}+\omega_{18}$ \\
$\omega_{5} \equiv \alpha$ & $\omega_{13} \equiv \frac{\lambda^{b}\left[1-\delta^{h}+B \theta e^{\theta}\right]}{\omega_{11}+\omega_{12}}$ & $\omega_{21}=\omega_{13}-\omega_{14}$ \\
$\omega_{6} \equiv \frac{B \theta e^{\theta}}{n \gamma}$ & $\omega_{14} \equiv \frac{B \lambda^{b} \theta e^{\theta}}{\omega_{11}+\omega_{12}}$ & $\omega_{22}=\omega_{10} \omega_{13}+\omega_{15}$ \\
$\omega_{7} \equiv \frac{B e^{\theta}}{n \gamma}$ & $\omega_{15} \equiv \frac{B \lambda^{b} \theta^{2} e^{\theta}}{\omega_{11}+\omega_{12}}$ & $\omega_{23}=\omega_{10}+\omega_{6} \omega_{17}$ \\
$\omega_{8} \equiv \sigma$ & $\omega_{16} \equiv \frac{\sigma \omega_{11}}{\omega_{11}+\omega_{12}}$ & $\omega_{24}=\omega_{7} \omega_{17}-1$ \\
\hline
\end{tabular}




\subsection{Log-marginal likelihood difference test}

The Bayes factors for comparing the models are given by the ratio of the two marginal likelihoods for $M_{H C}$ and $M_{R B C}$,

$$
B F_{H C, R B C}=\frac{p\left(\mathbf{y} \mid M_{H C}\right)}{p\left(\mathbf{y} \mid M_{R B C}\right)} .
$$

The marginal likelihood identity follows from Bayes' formula:

$$
\begin{aligned}
p\left(\boldsymbol{\psi}_{j} \mid \mathbf{y}, M_{j}\right) & =\frac{p\left(\mathbf{y} \mid \boldsymbol{\psi}_{j}, M_{j}\right) p\left(\boldsymbol{\psi}_{j} \mid M_{j}\right)}{p\left(\mathbf{y} \mid M_{j}\right)} \\
p\left(\mathbf{y} \mid M_{j}\right) & =\frac{p\left(\mathbf{y} \mid \boldsymbol{\psi}_{j}, M_{j}\right) p\left(\boldsymbol{\psi}_{j} \mid M_{j}\right)}{p\left(\boldsymbol{\psi}_{j} \mid \mathbf{y}, M_{j}\right)}, j=H C, R B C .
\end{aligned}
$$

Calculated at e.g. the mean of the posterior density $\boldsymbol{\psi}_{j}^{\star}$, the logarithm of the marginal likelihood is

$\ln p\left(\mathbf{y} \mid M_{j}\right)=\ln p\left(\mathbf{y} \mid \boldsymbol{\psi}_{j}^{\star}, M_{j}\right)+\ln p\left(\boldsymbol{\psi}^{\star} \mid M_{j}\right)-\ln p\left(\boldsymbol{\psi}_{j}^{\star} \mid \mathbf{y}, M_{j}\right), j=H C, R B C$.

To compute the marginal likelihood, we need to find $p\left(\boldsymbol{\psi}_{j}^{\star} \mid \mathbf{y}, M_{j}\right)$. For the $\mathrm{RBC}$ model, the procedure is straightforward: we denote the candidate generating density for the move from $\boldsymbol{\psi}$ to $\boldsymbol{\psi}^{\prime}$ as $q\left(\boldsymbol{\psi}^{\prime} \mid \boldsymbol{\psi}, \mathbf{y}\right)$. The acceptance probability is given as

$$
\begin{aligned}
p\left(\boldsymbol{\psi}^{\star} \mid \boldsymbol{\psi}, \mathbf{y}\right) & =\alpha\left(\boldsymbol{\psi}^{\star} \mid \boldsymbol{\psi}, \mathbf{y}\right) q\left(\boldsymbol{\psi}^{\star} \mid \boldsymbol{\psi}, \mathbf{y}\right) \\
\alpha\left(\boldsymbol{\psi}^{\star} \mid \boldsymbol{\psi}, \mathbf{y}\right) & =\min \left(1, \frac{f\left(\mathbf{y} \mid \boldsymbol{\psi}^{\star}\right) p\left(\boldsymbol{\psi}^{\star}\right)}{f(\mathbf{y} \mid \boldsymbol{\psi}) p(\boldsymbol{\psi})} \frac{q\left(\boldsymbol{\psi} \mid \boldsymbol{\psi}^{\star}, \mathbf{y}\right)}{q\left(\boldsymbol{\psi}^{\star} \mid \boldsymbol{\psi}, \mathbf{y}\right)}\right) .
\end{aligned}
$$

Integrating

$$
p\left(\boldsymbol{\psi}^{\star} \mid \boldsymbol{\psi}, \mathbf{y}\right) p(\boldsymbol{\psi} \mid \mathbf{y})=p\left(\boldsymbol{\psi} \mid \boldsymbol{\psi}^{\star}, \mathbf{y}\right) p\left(\boldsymbol{\psi}^{\star} \mid \mathbf{y}\right)
$$

over $\boldsymbol{\psi}$, we obtain

$$
p\left(\boldsymbol{\psi}^{\star} \mid \mathbf{y}\right)=\frac{\int \alpha\left(\boldsymbol{\psi}^{\star} \mid \boldsymbol{\psi}, \mathbf{y}\right) q\left(\boldsymbol{\psi}^{\star} \mid \boldsymbol{\psi}, \mathbf{y}\right) p(\boldsymbol{\psi} \mid \mathbf{y}) \mathrm{d} \boldsymbol{\psi}}{\int \alpha\left(\boldsymbol{\psi} \mid \boldsymbol{\psi}^{\star}, \mathbf{y}\right) q\left(\boldsymbol{\psi} \mid \boldsymbol{\psi}^{\star}, \mathbf{y}\right) \mathrm{d} \boldsymbol{\psi}}
$$

which is a ratio of two expected values

$$
p\left(\boldsymbol{\psi}^{\star} \mid \mathbf{y}\right)=\frac{\mathrm{E}\left(\alpha\left(\boldsymbol{\psi}^{\star} \mid \boldsymbol{\psi}, \mathbf{y}\right) q\left(\boldsymbol{\psi}^{\star} \mid \boldsymbol{\psi}, \mathbf{y}\right)\right)}{\mathrm{E}\left(\alpha\left(\boldsymbol{\psi} \mid \boldsymbol{\psi}^{\star}, \mathbf{y}\right)\right)}
$$

that can be estimated as

$$
\hat{p}\left(\boldsymbol{\psi}^{\star} \mid \mathbf{y}\right)=\frac{\frac{1}{S} \sum_{s=1}^{S} \alpha\left(\boldsymbol{\psi}^{\star} \mid \boldsymbol{\psi}_{s}, \mathbf{y}\right) q\left(\boldsymbol{\psi}^{\star} \mid \boldsymbol{\psi}_{s}, \mathbf{y}\right)}{\frac{1}{J} \sum_{j=1}^{J} \alpha\left(\boldsymbol{\psi}_{j} \mid \boldsymbol{\psi}^{\star}, \mathbf{y}\right)}
$$


where $\boldsymbol{\psi}_{s}$ are realizations of $\boldsymbol{\psi}$ from the posterior distribution and $\boldsymbol{\psi}_{j}$ are draws from the candidate generating density conditional on $\boldsymbol{\psi}^{\star}$. Substituting $\hat{p}\left(\boldsymbol{\psi}^{\star} \mid \mathbf{y}\right)$ into the logarithm of the marginal likelihood identity gives

$$
\begin{aligned}
\ln \hat{p}\left(\mathbf{y} \mid M_{R B C}\right) & =\ln p\left(\mathbf{y} \mid \boldsymbol{\psi}_{j}^{\star}, M_{R B C}\right)+\ln p\left(\boldsymbol{\psi}^{\star} \mid M_{R B C}\right)- \\
& -\ln \hat{p}\left(\boldsymbol{\psi}_{R B C}^{\star} \mid \mathbf{y}, M_{R B C}\right) .
\end{aligned}
$$

Since the parameters for the human capital model are generated using a twoblock Metropolis-Hastings algorithm, we follow the procedure described in Chib and Jeliatzkov (2001, p. 272-273). The marginal likelihood is given as

$$
\begin{aligned}
\ln \hat{p}\left(\mathbf{y} \mid M_{H C}\right) & =\ln p\left(\mathbf{y} \mid \boldsymbol{\psi}_{j}^{\star}, M_{H C}\right)+\ln p\left(\boldsymbol{\psi}^{\star} \mid M_{H C}\right)- \\
& -\ln \hat{p}\left(\boldsymbol{\psi}_{H C, 1}^{\star} \mid \mathbf{y}, M_{H C}\right)-\ln \hat{p}\left(\boldsymbol{\psi}_{H C, 2}^{\star} \mid \mathbf{y}, M_{H C}, \boldsymbol{\psi}_{H C, 1}^{\star}\right),
\end{aligned}
$$

where $\boldsymbol{\psi}_{H C, 1}^{\star}$ is the first parameter block (i.e. the de-trender), and $\boldsymbol{\psi}_{H C, 2}^{\star}$ contains the rest of the parameters.

\subsection{Multi-move Gibbs sampling}

In our case, we divide the vector of unknowns in two blocks: the state variables $\boldsymbol{\alpha}_{t}, t=1, \ldots, T$ and the vector with hyper-parameters $\boldsymbol{\psi}$. Following Carter and Kohn (1994), Gibbs sampling can be implemented to find $\mathbf{A}_{1, \ldots, T}$ from the joint distribution $p\left(\mathbf{A}_{1, \ldots, T} \mid \mathbf{Y}_{1, \ldots, T}\right)$, where

$$
\mathbf{A}_{1, \ldots, T}=\left(\begin{array}{llll}
\boldsymbol{\alpha}_{1}^{\prime} & \boldsymbol{\alpha}_{2}^{\prime} & \ldots & \boldsymbol{\alpha}_{T}^{\prime}
\end{array}\right)^{\prime}, \mathbf{Y}_{1, \ldots, T}=\left(\begin{array}{llll}
\mathbf{y}_{1}^{\prime} & \mathbf{y}_{2}^{\prime} & \ldots & \mathbf{y}_{T}^{\prime}
\end{array}\right)^{\prime} .
$$

The Gibbs sampler has two steps:

1. Generate the states $\mathbf{A}_{1, \ldots, T}$ conditional on the hyper-parameters $\boldsymbol{\psi}$ and the data $\mathbf{Y}_{1, \ldots, T}$.

2. Generate $\boldsymbol{\psi}$ conditional on $\mathbf{A}_{1, \ldots, T}$ and $\mathbf{Y}_{1, \ldots, T}$.

Because $\boldsymbol{\alpha}_{t}$ follows a Markov process, the joint distribution $p\left(\mathbf{A}_{1, \ldots, T} \mid \mathbf{Y}_{1, \ldots, T}\right)$ can be decomposed as

$$
p\left(\mathbf{a}_{T} \mid \mathbf{Y}_{1, \ldots, T}\right)=p\left(\boldsymbol{\alpha}_{T} \mid \mathbf{Y}_{1, \ldots, T}\right) \prod_{t=1}^{T-1} p\left(\boldsymbol{\alpha}_{t} \mid \boldsymbol{\alpha}_{t+1}, \mathbf{Y}_{1 \ldots, t}\right)
$$

To generate $\boldsymbol{\alpha}_{T}$ conditional on the data $\mathbf{Y}_{1, \ldots, T}$, observe that $\boldsymbol{\alpha}_{T}$ is normally distributed with expected value $\mathbf{a}_{T}$ and variance covariance matrix $\mathbf{P}_{T}$. To derive $\boldsymbol{a}_{T}$ and $\mathbf{P}_{T}$, the Kalman filter can be used. 
With the last step of the filter, we obtain $\boldsymbol{a}_{T}$ and $\boldsymbol{P}_{T}$, and a realization $\boldsymbol{\alpha}_{T}$ can be generated from

$$
\boldsymbol{\alpha}_{T} \mid \mathbf{Y}_{1, \ldots, T} \sim N\left(\mathbf{a}_{T}, \mathbf{P}_{T}\right)
$$

To obtain $\boldsymbol{\alpha}_{T-1} \mid \boldsymbol{\alpha}_{T}, \mathbf{Y}_{1, \ldots, T-1}$, note that the expected value of $\boldsymbol{\alpha}_{T-1}$ conditional on the observations $\mathbf{y}_{1}, \ldots, \mathbf{y}_{T-1}$ is given by ${ }^{27}$

$$
E\left[\boldsymbol{\alpha}_{T-1} \mid \mathbf{y}_{1}, \ldots, \mathbf{y}_{T-1}\right]=\mathbf{a}_{T-1},
$$

derived in the updating step of the filter. The expected value of $\boldsymbol{\alpha}_{T}$ conditional on $\mathbf{y}_{1}, \ldots, \mathbf{y}_{T-1}$ comes from the prediction step of the filter:

$$
E\left[\boldsymbol{\alpha}_{T} \mid \mathbf{y}_{1}, \ldots, \mathbf{y}_{T-1}\right]=\mathbf{a}_{T \mid T-1}=\mathbf{c}+\mathbf{T} \mathbf{a}_{T-1} .
$$

Therefore,

$$
\left(\begin{array}{c}
\boldsymbol{\alpha}_{T-1} \\
\boldsymbol{\alpha}_{T}
\end{array}\right) \sim N\left(\left(\begin{array}{c}
\mathbf{a}_{T-1} \\
\mathbf{c}+\mathbf{T a}_{T-1}
\end{array}\right),\left(\begin{array}{cc}
\mathbf{P}_{T-1} & \mathbf{P}_{T-1} \mathbf{T}^{\prime} \\
\mathbf{T P}_{T-1} & \mathbf{T P}_{T-1} \mathbf{T}^{\prime}+\mathbf{R Q R}^{\prime}
\end{array}\right)\right)
$$

The expected value of $\boldsymbol{\alpha}_{T-1}$ conditional on $\boldsymbol{\alpha}_{T}$ is

$$
\begin{aligned}
\mathbf{L}_{T-1} & =\mathbf{P}_{T-1} \mathbf{T}^{\prime}\left(\mathbf{T P}_{T-1} \mathbf{T}^{\prime}+\mathbf{R Q R} \mathbf{R}^{\prime}\right)^{-1} ; \\
\mathbf{a}_{T-1 \mid T} & =\mathbf{a}_{T-1}+\mathbf{L}_{T-1}\left(\boldsymbol{\alpha}_{T}-\mathbf{c}-\mathbf{T} \mathbf{a}_{T-1}\right) .
\end{aligned}
$$

and for the conditional covariance matrix we get

$$
\mathbf{P}_{T-1 \mid T}=\mathbf{P}_{T-1}-\mathbf{L}_{T-1} \mathbf{T P}_{T-1} .
$$

In general, $\boldsymbol{\alpha}_{t} \mid \boldsymbol{\alpha}_{t+1}, \mathbf{Y}_{1, \ldots, t}$ can be generated from

$$
\boldsymbol{\alpha}_{t} \mid \boldsymbol{\alpha}_{t+1}, \mathbf{Y}_{1, \ldots, t} \sim N\left(\mathbf{a}_{t \mid t+1}, \mathbf{P}_{t \mid t+1}\right),
$$

where

$$
\begin{aligned}
\mathbf{L}_{t} & =\mathbf{P}_{t} \mathbf{T}^{\prime}\left(\mathbf{T P}_{t} \mathbf{T}^{\prime}+\mathbf{R Q R}\right)^{-1} \\
\mathbf{a}_{t \mid t+1} & =\mathbf{a}_{t}+\mathbf{L}_{t}\left(\boldsymbol{\alpha}_{t+1}-\mathbf{c}-\mathbf{T} \mathbf{a}_{t}\right) ; \\
\mathbf{P}_{t \mid t+1} & =\mathbf{P}_{t}-\mathbf{L}_{t} \mathbf{T} \mathbf{P}_{t} .
\end{aligned}
$$

\footnotetext{
${ }^{27}$ For the following, see Harvey (1992, p. 154) and Hamilton (1994, p. 394-397).
} 